\title{
Positive periodic solutions and optimal control for a distributed biological model of two interacting species
}

\author{
G. Fragnelli ${ }^{1}$, P. Nistri ${ }^{1}$, D. Papini*,1 \\ ${ }^{a}$ Dipartimento di Ingegneria dell'Informazione, Università degli Studi di Siena \\ via Roma 56, 53100 Siena, Italy
}

\begin{abstract}
The paper deals with the existence of positive periodic solutions to a system of degenerate parabolic equations with delayed nonlocal terms and Dirichlet boundary conditions. Taking in each equation a meaningful function as a control parameter, we show that for a suitable choice of a class of such controls we have, for each of them, a time-periodic response of the system under different assumptions on the kernels of the nonlocal terms. Finally, we consider the problem of the minimization of a cost functional on the set of pairs: controlperiodic response. The considered system may be regarded as a possible model for the coexistence problem of two biological populations, which dislike crowding and live in a common territory, under different kind of intra- and inter-specific interferences.
\end{abstract}

Key words: Degenerate parabolic equations, nonlocal delayed terms, periodic solutions, topological degree theory, optimal control problem 2009 MSC: Primary: 35K65, 49J20; Secondary: 35B10, 47H11

\section{Introduction}

We consider the following system of degenerate parabolic equations with delayed nonlocal terms on $Q_{T}=\Omega \times(0, T)$ with periodic and Dirichlet boundary conditions:

$$
\left\{\begin{array}{l}
\frac{\partial u}{\partial t}-\Delta u^{m}=\left(a(x, t)-\int_{\Omega} K_{1}(\xi, t) u^{2}\left(\xi, t-\tau_{1}\right) d \xi+\int_{\Omega} K_{2}(\xi, t) v^{2}\left(\xi, t-\tau_{2}\right) d \xi\right) u \\
\frac{\partial v}{\partial t}-\Delta v^{m}=\left(b(x, t)+\int_{\Omega} K_{3}(\xi, t) u^{2}\left(\xi, t-\tau_{3}\right) d \xi-\int_{\Omega} K_{4}(\xi, t) v^{2}\left(\xi, t-\tau_{4}\right) d \xi\right) v \\
\left.u(\cdot, t)\right|_{\partial \Omega}=\left.v(\cdot, t)\right|_{\partial \Omega}=0, \quad \text { for } t \in[0, T] \\
u(\cdot, 0)=u(\cdot, T) \text { and } v(\cdot, 0)=v(\cdot, T)
\end{array}\right.
$$

\footnotetext{
${ }^{*}$ Corresponding author

Email addresses: fragnelli@dii.unisi.it (G. Fragnelli), pnistri@dii.unisi.it (P. Nistri), papini@di.unisi.it (D. Papini)
} 
and we look for continuous weak solutions. Here $\tau_{i} \in(0,+\infty), m>1, s^{m}=$ $|s|^{m-1} s, \Omega$ is a bounded domain of $\mathbb{R}^{n}$ with smooth boundary $\partial \Omega$, and $K_{i}, a, b \in$ $L^{\infty}\left(Q_{T}\right)$ are extended to $\Omega \times \mathbb{R}$ by $T$-periodicity.

Degenerate parabolic equations like those in system (1) model nonlinear diffusive phenomena and have been the subject of extensive study (see the recent monographs [8], [21] and their bibliography). In particular, system (1) is a possible model for the evolution of two biological species living in a common territory $\Omega$ where $u(x, t)$ and $v(x, t)$ are the respective densities of population at time $t$ located at $x \in \Omega$, while $a(x, t)$ and $b(x, t)$ are the growth rates at $x$ and time $t$ of the two populations in absence of any intra- and inter- interferences. In this framework the nonlinear terms $\Delta u^{m}$ and $\Delta v^{m}, m>1$, are proposed for instance in [10], [11], [18] and [20] instead of $\Delta u$ and $\Delta v$ in order to represent the tendency of the populations to avoid crowding. The nonlocal terms $\int_{\Omega} K_{i}(\xi, t) u^{2}\left(\xi, t-\tau_{i}\right) d \xi$ and $\int_{\Omega} K_{i}(\xi, t) v^{2}\left(\xi, t-\tau_{i}\right) d \xi$ evaluate a weighted fraction of individuals that actually interact at time $t>0$. The choice of considering $u^{2}, v^{2}$ in the nonlocal terms is mainly motivated by our approach that, as in [13], requires a priori estimates in $L^{2}$-norm for the solution of the regularized problem obtained from (1) by adding $-\epsilon \Delta u, \epsilon>0$, to the left hand sides of the two differential equations. The functions $K_{1}, K_{4}$ are supposed to be nonnegative and, roughly speaking, they measure the competition for food among each specie. On the other hand $K_{2}, K_{3}$ model the influence of a population on the other one. Observe that the system is cooperative if $K_{2}, K_{3}$ are non-negative and it is competitive if they are non-positive. We also consider cases where $K_{2}, K_{3}$ change sign in $Q_{T}$. The delayed densities $u, v$ at time $t-\tau_{i}$, that appear in the nonlocal terms, take into account the time needed to an individual to become adult and, thus, to interact and compete. Therefore, the term on the right hand side of each equation in (1) denotes the actual increasing rate of the population at $(x, t) \in Q_{T}$. We remark that we have $u(\cdot, s)=u(\cdot, s+T)$ and $v(\cdot, s)=v(\cdot, s+T)$ for $s \in(-\tau, 0)$, whenever $\tau>0$, since we are looking for $T$-periodic solutions $u, v$ to (1).

The interest in studying the existence of positive periodic solutions for reaction diffusion equations that model biological and physical phenomena, relies in the consideration that the periodic behavior of certain biological and physical non-negative quantities is the most natural and desirable one, see e.g. [1], [2], $[3],[4],[12],[13],[14],[17],[18]$ and [23]. For this reason we look at the functions $a, b$ as control parameters, i.e. we assume that the intrinsic growth rates $a, b$ can be modified by means of an external action at a certain time $t>0$ and at a certain location $x \in \Omega$. In this paper, under different assumption on the kernels $K_{i}$ of the nonlocal terms, we are interested in determining a class of essentially bounded functions $a, b$ in order to have for each pair of them a $T$-periodic continuous weak solution $(u, v)$ to (1) with $u, v$ non-negative and non-trivial in $Q_{T}$. Moreover, we aim at minimizing a given cost functional associated to (1) on the set $(u, v, a, b)$. In this framework the controls represent the intrinsic growth rates of the two populations.

A similar optimization problem has been considered in [2] for a single reac- 
tion diffusion equation of the form:

$$
\frac{\partial u}{\partial t}-\Delta u=f(x, t, \Phi(u), u, a) u,
$$

where $\Phi(u)$ is a coercive and positive nonlocal term, i.e. $\Phi(u) \geq C\|u(t)\|_{L^{1}(\Omega)}$, and $a=a(x, t)$ belongs to a suitable set $U \subset L^{\infty}\left(Q_{T}\right)$ of controls, $f$ satisfies the following growth and positivity conditions:

$\left(\mathrm{H}_{1}\right)$ : for any given $a \in U$ there exists $M \in L^{\infty}\left(Q_{T}\right)$ such that

$$
f(x, t, r, s, a(x, t)) \leq M(x, t)-\eta r
$$

for some positive constant $\eta$ if $r, s$ are nonnegative;

$\left(\mathrm{H}_{2}\right)$ : for any given $a \in U$ we have

$$
\frac{1}{T} \int_{0}^{T} f(x, t, \Phi(u), u(x, t), a(x, t)) d t>\mu_{1} \quad \text { for } \quad \text { a.a. } x \in \Omega
$$

if $0 \leq u \in C^{\alpha}\left(Q_{T}\right)$ and $\|u\|_{\infty}$ is sufficiently small. Here $\mu_{1}$ denotes the least eigenvalue of $-\Delta$ in $\Omega$ with Dirichlet boundary conditions.

The authors of [2] pointed out that monotonicity methods fail here due to the presence of the nonlocal term and thus cannot be employed to prove the existence of periodic solutions of (2) (see [2, Theorem 0]). In fact they adopted a topological approach based on a priori bounds and the continuation property of Leray-Schauder topological degree.

Recently these results and the relative techniques have been extended in [14] to the following degenerate equation:

$$
\frac{\partial u}{\partial t}-\Delta u^{m}=(a(x, t)-\Phi(u)) u, \quad \text { in } Q_{T} \text { for } m>1,
$$

with $T$-periodic and Dirichlet boundary conditions. Here $\Phi: L^{2}(\Omega)^{+} \rightarrow \mathbb{R}^{+}$is a bounded, continuous and coercive functional, i.e.:

$$
C_{1}\|w\|_{L^{2}(\Omega)}^{2} \leq \Phi(w) \leq C_{2}\|w\|_{L^{2}(\Omega)}^{2},
$$

where $w(\cdot)=u(\cdot, t), C_{1}, C_{2}$ are positive constants, and $L^{2}(\Omega)^{+}:=\left\{w \in L^{2}(\Omega)\right.$ : $w \geq 0$, a.e. in $\Omega\}$. On the other hand $a(x, t)$ satisfies

$$
\underset{x \in \Omega}{\operatorname{essinf}} \frac{1}{T} \int_{0}^{T} a(x, t) d t>\mu_{1},
$$

in agreement with assumption $\left(\mathrm{H}_{2}\right)$ of [2].

The literature about systems of degenerate parabolic equations is scarce and, to our knowledge, there are no papers dealing with the periodic problem (for systems of nondegenerate parabolic equations see, for example, [9]). In this paper we consider the problem of the existence of a continuous $T$-periodic weak 
solution $(u, v)$ of problem (1) with non-trivial $u, v \geq 0$ in $Q_{T}$, where the growth rates $a, b$ and the functions $K_{i}$ are chosen in the class $L^{\infty}\left(Q_{T}\right)$ and must satisfy conditions involving $\mu_{1}$.

More precisely, a first existence result, Theorem 3.1, is obtained by assuming the coercivity in $L^{2}(\Omega)$ of the nonlocal terms corresponding to $K_{1}$ and $K_{4}$ and using suitable bounds on $K_{2}$ and $K_{3}$. As already noticed, for a single equation a coercivity assumption was considered in both [2] and [14]. In particular, competitive systems (those with $K_{2} \leq 0$ and $K_{3} \leq 0$ a.e. in $Q_{T}$ ) and cooperative systems (those with $K_{2} \geq 0$ and $K_{3} \geq 0$ a.e. in $Q_{T}$ ) fall into this existence result.

Furthermore, two existence results are proved in Theorem 3.2 and Theorem 3.3 for competitive systems without assuming the coercivity in $L^{2}(\Omega)$ of the nonlocal terms corresponding to $K_{1}$ and $K_{4}$.

An existence result is finally obtained in Theorem 3.4 without that coercivity and any sign condition on $K_{2}, K_{3}$ by adding the technical assumption $m>3$ in the degenerate terms $\Delta u^{m}$ and $\Delta v^{m}$.

We follow a standard technique to deal with the degeneracy of the equations (see for instance [4], [14], [17]) by perturbing the degenerate part of the equations $\Delta u^{m}$ and $\Delta v^{m}$ by $\epsilon \Delta u$ and $\epsilon \Delta v$ respectively, with $\epsilon>0$ small enough. We thus obtain a family of regularized non-degenerate problems and we solve them by means of the topological degree theory. We finally get a solution of (1) passing to the limit as $\epsilon \rightarrow 0$. This procedure is carried out once for all in Section 2 and, in particular, in Theorem 2.1 where we show that the explicit knowledge of a priori bounds for the $L^{2}$-norms of the solutions of the regularized problems and the positivity of a certain value $\eta$, that depends on the bounds, are sufficient to prove the existence of a non-negative solution $(u, v)$ of (1) with non-trivial $u, v$ (i.e. periodic coexistence). We devote Section 3 to finding the required a priori bounds and to state the definitive existence results and their corollaries in some significative situations. Finally, in Section 4, we introduce a cost functional $J(u, v, a, b)$ to be minimized on the set of all the quadruple $(u, v, a, b)$, where $u, v$ is the $T$-periodic solution to (1) corresponding to $a, b$. The considered cost functional is suitable to evaluate the difference between the cost of controlling the growth rate to the values $a(x, t), b(x, t)$ and the benefits due to the presence of the two populations of density $u(x, t)$ and $v(x, t)$ respectively.

Notation. Throughout the paper $\Omega \subset \mathbb{R}^{n}$ is an open and bounded set with smooth boundary $\partial \Omega$ and $Q_{T}=\Omega \times[0, T]$, for $T>0 ;\|u\|_{p}$ denotes the usual norm in $L^{p}\left(Q_{T}\right)$ for $1 \leq p \leq \infty ; \mu_{1}$ denotes the first eigenvalue of $-\Delta$ on $\Omega$ with homogeneous Dirichlet boundary condition and $\phi_{1}$ is the corresponding positive eigenfunction with $\left\|\phi_{1}\right\|_{L^{2}(\Omega)}=1$. We always adopt the notation $s^{m}=|s|^{m-1} s$ for any $m>1$ and $s \in \mathbb{R}$.

\section{Preliminary results and a general coexistence theorem}

Throughout the paper we will assume that $a, b, K_{i} \in L^{\infty}\left(Q_{T}\right)$ in (1). We now recall the definition of a weak solution to (1). 
Definition 2.1. A pair of functions $(u, v)$ is said to be a weak solution of (1) if $u, v \in C\left(\bar{Q}_{T}\right), u^{m}, v^{m} \in L^{2}\left((0, T) ; H_{0}^{1}(\Omega)\right)$ and $(u, v)$ satisfies

$$
\begin{gathered}
\iint_{Q_{T}}\left(-u \frac{\partial \varphi}{\partial t}+\nabla u^{m} \nabla \varphi-a u \varphi+u \varphi \int_{\Omega}\left[K_{1}(\xi, t) u^{2}\left(\xi, t-\tau_{1}\right)-\right.\right. \\
\left.\left.-K_{2}(\xi, t) v^{2}\left(\xi, t-\tau_{2}\right)\right] d \xi\right) d x d t=0
\end{gathered}
$$

and

$$
\begin{gathered}
\iint_{Q_{T}}\left(-v \frac{\partial \varphi}{\partial t}+\nabla v^{m} \nabla \varphi-b v \varphi+v \varphi \int_{\Omega}\left[-K_{3}(\xi, t) u^{2}\left(\xi, t-\tau_{3}\right)+\right.\right. \\
\left.\left.+K_{4}(\xi, t) v^{2}\left(\xi, t-\tau_{4}\right)\right] d \xi\right) d x d t=0
\end{gathered}
$$

for any $\varphi \in C^{1}\left(\bar{Q}_{T}\right), \varphi(x, T)=\varphi(x, 0)$ for any $x \in \Omega$ and $\varphi(x, t)=0$ for any $(x, t) \in \partial \Omega \times[0, T]$.

Here and in the following we assume that the functions $t \rightarrow u(\cdot, t)$ and $t \rightarrow v(\cdot, t)$ are extended from $[0, T]$ to $\mathbb{R}$ by $T$-periodicity so that $(u, v)$ is a solution for all $t$.

Due to the degeneracy of the equation we consider, as in [17] and [14], the following regularized (non-degenerate) problem:

$$
\left\{\begin{aligned}
& \frac{\partial u}{\partial t}-\Delta\left(u^{m}+\epsilon u\right)=\left(a(x, t)-\int_{\Omega} K_{1}(\xi, t) u^{2}\left(\xi, t-\tau_{1}\right) d \xi+\right. \\
&\left.+\int_{\Omega} K_{2}(\xi, t) v^{2}\left(\xi, t-\tau_{2}\right) d \xi\right) u \\
& \frac{\partial v}{\partial t}-\Delta\left(v^{m}+\epsilon v\right)=\left(b(x, t)+\int_{\Omega} K_{3}(\xi, t) u^{2}\left(\xi, t-\tau_{3}\right) d \xi-\right. \\
&\left.-\int_{\Omega} K_{4}(\xi, t) v^{2}\left(\xi, t-\tau_{4}\right) d \xi\right) v \\
&\left.u(\cdot, t)\right|_{\partial \Omega}=\left.v(\cdot, t)\right|_{\partial \Omega}=0, \quad \text { for a.a. } t \in(0, T), \\
& u(\cdot, 0)=u(\cdot, T) \text { and } v(\cdot, 0)=v(\cdot, T)
\end{aligned}\right.
$$

where $(x, t) \in Q_{T}$ and $\epsilon \in(0,1 / 2)$. A solution $(u, v)$ of (1) will be then obtained as the limit, for $\epsilon \rightarrow 0$, of the solutions $\left(u_{\epsilon}, v_{\epsilon}\right)$ of (3), which will be functions in $L^{2}\left((0, T) ; H_{0}^{1}(\Omega)\right) \cap C\left(\bar{Q}_{T}\right)$ satisfying (3) in the usual weak sense.

To deal with the existence of $T$-periodic solutions $\left(u_{\epsilon}, v_{\epsilon}\right)$ of system (3), with $u_{\epsilon}, v_{\epsilon} \geq 0$ in $Q_{T}$, we introduce, for any $\epsilon \in(0,1 / 2)$, the map $G_{\epsilon}:[0,1] \times$ $L^{\infty}\left(Q_{T}\right) \times L^{\infty}\left(Q_{T}\right) \rightarrow L^{\infty}\left(Q_{T}\right) \times L^{\infty}\left(Q_{T}\right)$ as follows:

$$
(\sigma, f, g) \mapsto\left(u_{\epsilon}, v_{\epsilon}\right)=G_{\epsilon}(\sigma, f, g)
$$


if and only if $\left(u_{\epsilon}, v_{\epsilon}\right)$ solves the following uncoupled problem

$$
\begin{cases}\frac{\partial u}{\partial t}-\Delta\left(\sigma u^{m}+\epsilon u\right)=f, & \text { for a.a. }(x, t) \in Q_{T}, \\ \frac{\partial v}{\partial t}-\Delta\left(\sigma v^{m}+\epsilon v\right)=g, & \text { for a.a. }(x, t) \in Q_{T}, \\ \left.u(\cdot, t)\right|_{\partial \Omega}=\left.v(\cdot, t)\right|_{\partial \Omega}=0, & \text { for a.a. } t \in(0, T), \\ u(\cdot, 0)=u(\cdot, T) \text { and } v(\cdot, 0)=v(\cdot, T) . & \end{cases}
$$

This map $G_{\epsilon}$ is well defined since [17, Theorem 2] grants existence and uniqueness for (4). Consider now

$$
f(p, q):=\left(a-\int_{\Omega} K_{1}(\xi, \cdot) p^{2}\left(\xi, \cdot-\tau_{1}\right) d \xi+\int_{\Omega} K_{2}(\xi, \cdot) q^{2}\left(\xi, \cdot-\tau_{2}\right) d \xi\right) p
$$

and

$$
g(p, q):=\left(b+\int_{\Omega} K_{3}(\xi, \cdot) p^{2}\left(\xi, \cdot-\tau_{3}\right) d \xi-\int_{\Omega} K_{4}(\xi, \cdot) q^{2}\left(\xi, \cdot-\tau_{4}\right) d \xi\right) q,
$$

where $p$ and $q$ belong to $L^{\infty}\left(Q_{T}\right)$. Clearly, if the nonnegative functions $u_{\epsilon}, v_{\epsilon} \in$ $L^{\infty}\left(Q_{T}\right)$ are such that $\left(u_{\epsilon}, v_{\epsilon}\right)=G_{\epsilon}\left(1, f\left(u_{\epsilon}, v_{\epsilon}\right), g\left(u_{\epsilon}, v_{\epsilon}\right)\right)$, then $\left(u_{\epsilon}, v_{\epsilon}\right)$ is also a solution of (3) (with $u_{\epsilon}$ and $v_{\epsilon} \geq 0$ ) in $Q_{T}$. Hence, the existence of a nonnegative solution of (3) is equivalent to the existence of a fixed point $(p, q)$ of the map $(p, q) \rightarrow G_{\epsilon}(1, f(p, q), g(p, q))$ with $p$ and $q \geq 0$.

As a matter of notation, throughout the paper we denote

$$
B_{R}=\left\{(p, q) \in L^{\infty}\left(Q_{T}\right) \times L^{\infty}\left(Q_{T}\right): \max \left\{\|p\|_{\infty},\|q\|_{\infty}\right\}<R\right\}, \quad R>0 .
$$

Let $T_{\epsilon}(\sigma, p, q):=G_{\epsilon}(\sigma, f(p, q), g(p, q))$, we have the following result.

Lemma 2.1. Let $(p, q) \in L^{\infty}\left(Q_{T}\right) \times L^{\infty}\left(Q_{T}\right)$ and let $\epsilon \in(0,1 / 2)$. Then $\left(u_{\epsilon}, v_{\epsilon}\right)=T_{\epsilon}(\sigma, p, q)$ is a compact continuous map from $[0,1] \times L^{\infty}\left(Q_{T}\right) \times$ $L^{\infty}\left(Q_{T}\right) \rightarrow L^{\infty}\left(Q_{T}\right) \times L^{\infty}\left(Q_{T}\right)$.

Proof. Let $(p, q) \in B$, where $B$ is a bounded set of $L^{\infty}\left(Q_{T}\right) \times L^{\infty}\left(Q_{T}\right)$, then $\{f(p, q):(p, q) \in B\}$ is a bounded set in $L^{\infty}\left(Q_{T}\right)$. The $T$-periodicity condition gives

$$
\left\|u_{\epsilon}(0, \cdot)\right\|_{L^{2}(\Omega)} \leq c\|f\|_{\infty},
$$

for some positive constant $c$. Since the initial data $u_{\epsilon}(0, \cdot)$ is bounded in $L^{2}(\Omega)$, it results

$$
\left\|u_{\epsilon}\right\|_{2} \leq c\|f\|_{\infty} .
$$

We may apply classical local estimates (see [15]) to bound $u_{\epsilon}$ in $L^{\infty}\left(\Omega \times\left[\frac{T}{2}, \frac{3 T}{2}\right]\right)$ and thus, by $T$-periodicity, for all $t$ in terms of $\|f\|_{\infty}$. Classical results in the above reference (Theorem 1.1 p. 419) also show that $u_{\epsilon} \in C^{\alpha, \alpha / 2}\left(Q_{T}\right)$ for some $\alpha>0$. Moreover, due to its uniform continuity in $Q_{T}, u_{\epsilon}$ can be uniquely extended by continuity to $\bar{Q}_{T}$. The same arguments apply to $v_{\epsilon}$, thus the pair 
$\left(u_{\epsilon}, v_{\epsilon}\right)$ satisfies $(4)$ and $u_{\epsilon}, v_{\epsilon} \in C\left(\bar{Q}_{T}\right)$. Finally, by the Ascoli-Arzelà Theorem, the compactness and continuity of $T_{\epsilon}$ follow from the bound in the $L^{\infty}$-norm of $u_{\epsilon}$ and $v_{\epsilon}$ and the fact that they are equi-Hölder continuous in $\bar{Q}_{T}([15$, Theorem 1.1]).

Our aim is to prove the existence of $T$-periodic solutions $u_{\epsilon}, v_{\epsilon} \in C\left(\bar{Q}_{T}\right)$, $u_{\epsilon}, v_{\epsilon}>0$ in $Q_{T}$, of the regularized problem (3) for all $\epsilon>0$ small enough as positive fixed points of the map $(p, q) \rightarrow T_{\epsilon}(1, p, q)$. As a first step we prove the following result.

Proposition 2.1. Assume that $a, b, K_{i} \in L^{\infty}\left(Q_{T}\right)$ for $i=1,2,3,4$. If the nontrivial pair $\left(u_{\epsilon}, v_{\epsilon}\right)$ solves

$$
(u, v)=G_{\epsilon}\left(\sigma, \rho f\left(u^{+}, v^{+}\right)+(1-\sigma), \rho g\left(u^{+}, v^{+}\right)+(1-\sigma)\right),
$$

for some $\sigma \in[0,1]$ and $\rho \in[0,1]$, then

$$
u_{\epsilon}(x, t) \geq 0 \text { and } v_{\epsilon}(x, t) \geq 0 \quad \text { for any }(x, t) \in Q_{T} .
$$

Moreover, if $u_{\epsilon} \neq 0$ or $v_{\epsilon} \neq 0$ then $u_{\epsilon}>0$ or $v_{\epsilon}>0$ in $Q_{T}$, respectively.

Proof. Assume that $\left(u_{\epsilon}, v_{\epsilon}\right)$ solves (5) with $u_{\epsilon} \neq 0$ for some $\sigma \in[0,1]$ and $\rho \in[0,1]$. We first prove that $u_{\epsilon} \geq 0$. Multiplying the first equation of $(4)$, where $f(p, q)$ is replaced by $\rho f\left(u_{\epsilon}^{+}, v_{\epsilon}^{+}\right)+(1-\sigma)$, by $u_{\epsilon}^{-}:=\min \left\{0, u_{\epsilon}\right\}$, integrating on $Q_{T}$ and passing to the limit in the Steklov averages $\left(u_{\epsilon}\right)_{h} \in H^{1}\left(Q_{T-\delta}\right), \delta, h>0$, in the standard way $[15$, p. 85$]$, we obtain

$$
-\iint_{Q_{T}} \Delta\left(\sigma u_{\epsilon}^{m}+\epsilon u_{\epsilon}\right) u_{\epsilon}^{-} d x d t=\iint_{Q_{T}}(1-\sigma) u_{\epsilon}^{-} d x d t
$$

by the $T$-periodicity of $u_{\epsilon}$ and taking into account that $u_{\epsilon}^{+} u_{\epsilon}^{-}=0$. Hence we obtain

$$
-\iint_{Q_{T}} \Delta\left(\sigma u_{\epsilon}^{m}+\epsilon u_{\epsilon}\right) u_{\epsilon}^{-} d x d t=\iint_{Q_{T}} \nabla\left(\sigma u_{\epsilon}^{m}+\epsilon u_{\epsilon}\right) \nabla u_{\epsilon}^{-} d x d t \leq 0
$$

that is

$$
\iint_{Q_{T}} \sigma m\left|u_{\epsilon}^{-}\right|^{m-1}\left|\nabla u_{\epsilon}^{-}\right|^{2} d x d t+\epsilon \iint_{Q_{T}}\left|\nabla u_{\epsilon}^{-}\right|^{2} d x d t \leq 0,
$$

which implies that $u_{\epsilon}^{-}=0$ by Poincaré's inequality and, thus, $u_{\epsilon}=u_{\epsilon}^{+} \geq 0$ in $Q_{T}$.

Now we prove that $u_{\epsilon}>0$ in $Q_{T}$. Since $u_{\epsilon}$ is non-trivial, there exists $\left(x_{0}, t_{0}\right) \in \Omega \times(0, T]$ such that $u_{\epsilon}\left(x_{0}, t_{0}\right)>0$. Let $\psi \in C_{0}^{\infty}(\Omega)$ be a nonnegative function such that $0<\psi\left(x_{0}\right)<u_{\epsilon}\left(x_{0}, t_{0}\right)$ and, for $M>0$, let $z$ be a solution of

$$
\begin{cases}\frac{\partial z}{\partial t}-\Delta\left(\sigma z^{m}+\epsilon z\right)+M z=0, & (x, t) \in \Omega \times\left(t_{0}, t_{0}+T\right], \\ \left.z(\cdot, t)\right|_{\partial \Omega}=0, & \text { for } t \in\left[t_{0}, t_{0}+T\right] \\ z\left(\cdot, t_{0}\right)=\psi(\cdot) . & \end{cases}
$$


Since $a-\int_{\Omega} K_{1}(\xi, \cdot) u_{\epsilon}^{2}\left(\xi, \cdot-\tau_{1}\right) d \xi+\int_{\Omega} K_{2}(\xi, \cdot) v_{\epsilon}^{2}\left(\xi, \cdot-\tau_{2}\right) d \xi \in L^{\infty}\left(Q_{T}\right)$, we can choose $M$ large enough so that, by the comparison theorem,

$$
u_{\epsilon}(x, t) \geq z(x, t) \quad \text { for any }(x, t) \in \Omega \times\left[t_{0}, t_{0}+T\right] .
$$

By the maximum principle, $z(x, t)>0$ for any $(x, t) \in \Omega \times\left[t_{0}, t_{0}+T\right]$. Therefore, by $T$-periodicity, $u_{\epsilon}(x, t)>0$ for all $(x, t) \in Q_{T}$. In the same way, one can prove that $v_{\epsilon} \neq 0$ implies $v_{\epsilon}(x, t)>0$ for all $(x, t) \in Q_{T}$.

Remark 2.1. Observe that if $\rho=0$, by using the arguments of the proof of Proposition 2.1 to show that $u_{\epsilon}^{-}=0$, it can be shown that $(u, v)=G_{\epsilon}(1,0,0)$ if and only if $(u, v)=(0,0)$.

The following result guarantees that the solutions $\left(u_{\epsilon}, v_{\epsilon}\right)$ of $(3)$ we are going to find are not bifurcating from the trivial solution $(0,0)$ as $\epsilon$ ranges in $(0,1 / 2)$.

Proposition 2.2. Assume that

$$
\frac{1}{T} \iint_{Q_{T}} \phi_{1}^{2}(x) a(x, t) d x d t>\mu_{1} \quad \text { and } \quad \frac{1}{T} \iint_{Q_{T}} \phi_{1}^{2}(x) b(x, t) d x d t>\mu_{1}
$$

and let

$$
\begin{gathered}
r_{0}=\min \left\{\left(\frac{1}{2 m}\right)^{\frac{1}{m-1}},\left[\frac{\iint_{Q_{T}} \phi_{1}^{2}(x) a(x, t) d x d t-T \mu_{1}}{\left\|K_{1}\right\|_{1}+\left\|K_{2}\right\|_{1}}\right]^{1 / 2},\right. \\
\left.\left[\frac{\iint_{Q_{T}} \phi_{1}^{2}(x) b(x, t) d x d t-T \mu_{1}}{\left\|K_{3}\right\|_{1}+\left\|K_{4}\right\|_{1}}\right]^{1 / 2}\right\} .
\end{gathered}
$$

If the non-trivial pair $\left(u_{\epsilon}, v_{\epsilon}\right)$ solves $(u, v)=G_{\epsilon}\left(\sigma, f\left(u^{+}, v^{+}\right)+(1-\sigma), g\left(u^{+}, v^{+}\right)+\right.$ $(1-\sigma))$, for some $\sigma \in[0,1]$, then

$$
\max \left\{\left\|u_{\epsilon}\right\|_{\infty},\left\|v_{\epsilon}\right\|_{\infty}\right\} \geq r_{0} .
$$

Moreover $\operatorname{deg}\left((u, v)-T_{\epsilon}\left(1, u^{+}, v^{+}\right), B_{r}, 0\right)=0$ for all $r \in\left(0, r_{0}\right)$.

Proof. By contradiction, assume that for some $\sigma \in[0,1]$ and $r \in\left(0, r_{0}\right)$ there exists a pair $\left(u_{\epsilon}, v_{\epsilon}\right) \neq(0,0)$ such that $\left(u_{\epsilon}, v_{\epsilon}\right)=G_{\epsilon}\left(\sigma, f\left(u_{\epsilon}^{+}, v_{\epsilon}^{+}\right)+(1-\right.$ $\left.\sigma), g\left(u_{\epsilon}^{+}, v_{\epsilon}^{+}\right)+(1-\sigma)\right)$ with $\left\|u_{\epsilon}\right\|_{\infty} \leq r$ and $\left\|v_{\epsilon}\right\|_{\infty} \leq r$. Assume that $u_{\epsilon} \neq 0$ and take $\phi \in C_{0}^{\infty}(\Omega)$. Since by Proposition 2.1 we have $u_{\epsilon}>0$ in $Q_{T}$, we can multiply the equation

$$
\begin{aligned}
\frac{\partial u_{\epsilon}}{\partial t}-\Delta\left(\sigma u_{\epsilon}^{m}+\epsilon u_{\epsilon}\right)= & \left(a(x, t)-\int_{\Omega} K_{1}(\xi, t) u_{\epsilon}^{2}\left(\xi, t-\tau_{1}\right) d \xi+\right. \\
& \left.+\int_{\Omega} K_{2}(\xi, t) v_{\epsilon}^{2}\left(\xi, t-\tau_{2}\right) d \xi\right) u_{\epsilon}+(1-\sigma)
\end{aligned}
$$


by $\frac{\phi^{2}}{u_{\epsilon}}$, integrate over $Q_{T}$ and pass to the limit in the Steklov averages in order to obtain

$$
\begin{aligned}
-\iint_{Q_{T}} \frac{\phi^{2}}{u_{\epsilon}} \Delta\left(\sigma u_{\epsilon}^{m}+\epsilon u_{\epsilon}\right) d x d t & =\iint_{Q_{T}}\left(\phi^{2}(x) a(x, t)+(1-\sigma) \frac{\phi^{2}}{u_{\epsilon}}\right) d x d t- \\
& -\iint_{Q_{T}} \phi^{2}(x)\left(\int_{\Omega} K_{1}(\xi, t) u_{\epsilon}^{2}\left(\xi, t-\tau_{1}\right) d \xi\right) d x d t+ \\
& +\iint_{Q_{T}} \phi^{2}(x)\left(\int_{\Omega} K_{2}(\xi, t) v_{\epsilon}^{2}\left(\xi, t-\tau_{2}\right) d \xi\right) d x d t
\end{aligned}
$$

by the $T$-periodicity of $u_{\epsilon}$. Moreover, a straightforward computation shows that

$$
\begin{aligned}
-\iint_{Q_{T}} \frac{\phi^{2}}{u_{\epsilon}} \Delta\left(\sigma u_{\epsilon}^{m}+\epsilon u_{\epsilon}\right) d x d t & =\iint_{Q_{T}} \nabla\left(\frac{\phi^{2}}{u_{\epsilon}}\right) \nabla\left(\sigma u_{\epsilon}^{m}+\epsilon u_{\epsilon}\right) d x d t \\
& =\iint_{Q_{T}}\left(m \sigma u_{\epsilon}^{m-1}+\epsilon\right)\left(|\nabla \phi|^{2}-u_{\epsilon}^{2}\left|\nabla\left(\frac{\phi}{u_{\epsilon}}\right)\right|^{2}\right) d x d t .
\end{aligned}
$$

Since $r<\left(\frac{1}{2 m}\right)^{1 /(m-1)}$, then $m \sigma u_{\epsilon}^{m-1}+\epsilon \leq m u_{\epsilon}^{m-1}+\epsilon<1 / 2+\epsilon<1$ and

$$
\begin{aligned}
-\iint_{Q_{T}} \frac{\phi^{2}}{u_{\epsilon}} \Delta\left(\sigma u_{\epsilon}^{m}+\epsilon u_{\epsilon}\right) d x d t & \leq \iint_{Q_{T}}\left(m \sigma u_{\epsilon}^{m-1}+\epsilon\right)|\nabla \phi|^{2} d x d t \\
& <\iint_{Q_{T}}|\nabla \phi|^{2} d x d t .
\end{aligned}
$$

Hence, combining (7) and (8), we obtain

$$
\begin{aligned}
& \iint_{Q_{T}} \phi^{2}(x)\left(a(x, t)-\int_{\Omega} K_{1}(\xi, t) u_{\epsilon}^{2}\left(\xi, t-\tau_{1}\right) d \xi+\int_{\Omega} K_{2}(\xi, t) v_{\epsilon}^{2}\left(\xi, t-\tau_{2}\right) d \xi\right) d x d t \\
& <\iint_{Q_{T}}|\nabla \phi|^{2} d x d t .
\end{aligned}
$$

Taking $\phi(x)=\phi_{1}(x)$ one has

$$
\begin{aligned}
0< & \iint_{Q_{T}}\left[\left|\nabla \phi_{1}(x)\right|^{2}-\phi_{1}^{2}(x)\left(a(x, t)-\int_{\Omega} K_{1}(\xi, t) u_{\epsilon}^{2}\left(\xi, t-\tau_{1}\right) d \xi\right)\right] d x d t- \\
& -\iint_{Q_{T}}\left[\phi_{1}^{2}(x) \int_{\Omega} K_{2}(\xi, t) v_{\epsilon}^{2}\left(\xi, t-\tau_{2}\right) d \xi\right] d x d t \\
= & \mu_{1} T-\iint_{Q_{T}} \phi_{1}^{2}(x) a(x, t) d x d t+ \\
& +\iint_{Q_{T}}\left[K_{1}(\xi, t) u_{\epsilon}^{2}\left(\xi, t-\tau_{1}\right)-K_{2}(\xi, t) v_{\epsilon}^{2}\left(\xi, t-\tau_{2}\right)\right] d \xi d t
\end{aligned}
$$

and, by Hölder's inequality:

$$
\iint_{Q_{T}} \phi_{1}^{2}(x) a(x, t) d x d t-\mu_{1} T<\left\|K_{1}\right\|_{1} r^{2}+\left\|K_{2}\right\|_{1} r^{2} .
$$


Thus

$$
r_{0}^{2} \leq \frac{\iint_{Q_{T}} \phi_{1}^{2}(x) a(x, t) d x d t-\mu_{1} T}{\left\|K_{1}\right\|_{1}+\left\|K_{2}\right\|_{1}}<r^{2}
$$

that is a contradiction. The same argument applies if $v_{\epsilon} \neq 0$.

Let us now fix any $r \in\left(0, r_{0}\right)$. We just proved that

$$
(u, v) \neq G_{\epsilon}\left(\sigma, f\left(u^{+}, v^{+}\right)+(1-\sigma), g\left(u^{+}, v^{+}\right)+(1-\sigma)\right),
$$

$\forall(u, v) \in \partial B_{r}$ and $\forall \sigma \in[0,1]$. Hence the topological degree of $(u, v)-$ $G_{\epsilon}\left(\sigma, f\left(u^{+}, v^{+}\right)+(1-\sigma), g\left(u^{+}, v^{+}\right)+(1-\sigma)\right)$ is well defined in $B_{r}$ for all $\sigma \in[0,1]$. From the homotopy invariance of the Leray-Schauder degree, we have

$$
\begin{aligned}
& \operatorname{deg}\left((u, v)-T_{\epsilon}\left(1, u^{+}, v^{+}\right), B_{r}, 0\right) \\
& \quad=\operatorname{deg}\left((u, v)-G_{\epsilon}\left(0, f\left(u^{+}, v^{+}\right)+1, g\left(u^{+}, v^{+}\right)+1\right), B_{r}, 0\right)
\end{aligned}
$$

and the last degree is zero since the equation

$$
(u, v)=G_{\epsilon}\left(0, f\left(u^{+}, v^{+}\right)+1, g\left(u^{+}, v^{+}\right)+1\right)
$$

admits neither trivial nor non-trivial solutions in $B_{r}$.

The next result is our main tool to obtain coexistence results for (1). Roughly speaking, it says that the existence of non-negative non-trivial solutions $u, v$ of (1) follows as soon as we can check the positivity of a value $\eta\left(C_{1}, C_{2}\right)$, where $C_{1}, C_{2}$ are uniform a priori bounds on the $L^{2}$-norm of any solution pair $\left(u_{\epsilon}, v_{\epsilon}\right)$ of the regularized problem (3) for any $\epsilon>0$ small enough. The next section will be devoted to the search of such explicit bounds when something more is known on the structure of the equations.

Theorem 2.1. Assume that $K_{1}(x, t), K_{4}(x, t) \geq 0$ for a.a. $(x, t) \in Q_{T}$ and that there are $C_{1}, C_{2}>0$ such that

$$
\left\|u_{\epsilon}\right\|_{2}^{2} \leq C_{1} \text { and }\left\|v_{\epsilon}\right\|_{2}^{2} \leq C_{2}
$$

for all solution pairs $\left(u_{\epsilon}, v_{\epsilon}\right)$ of

$$
(u, v)=G_{\epsilon}\left(1, \rho f\left(u^{+}, v^{+}\right), \rho g\left(u^{+}, v^{+}\right)\right)
$$

and all $\epsilon \in(0,1 / 2)$ and $\rho \in(0,1]$. Then there is a constant $R>0$ such that

$$
\left\|u_{\epsilon}\right\|_{\infty},\left\|v_{\epsilon}\right\|_{\infty}<R
$$

for all solution pairs $\left(u_{\epsilon}, v_{\epsilon}\right)$ of $(11)$ and all $\epsilon \in(0,1 / 2)$ and $\rho \in(0,1]$. In particular, one has that

$$
\operatorname{deg}\left((u, v)-T_{\epsilon}\left(1, u^{+}, v^{+}\right), B_{R}, 0\right)=1 .
$$


Moreover, if

$$
\begin{aligned}
& \eta\left(C_{1}, C_{2}\right):=\min \{ \frac{1}{T} \iint_{Q_{T}} \phi_{1}^{2}(x) a(x, t) d x d t-\mu_{1}-\frac{\underline{k}_{2} C_{2}}{T}, \\
&\left.\frac{1}{T} \iint_{Q_{T}} \phi_{1}^{2}(x) b(x, t) d x d t-\mu_{1}-\frac{k_{3} C_{1}}{T}\right\}>0,
\end{aligned}
$$

where $\underline{k}_{2}, \underline{k}_{3}$ are such that $-\underline{k}_{2} \leq K_{2}(x, t)$ and $-\underline{k}_{3} \leq K_{3}(x, t)$ for a.a. $(x, t) \in$ $Q_{T}$, then problem (1) has a T-periodic non-negative solution $(u, v)$ with nontrivial $u, v$.

Proof. Assume $u_{\epsilon} \neq 0$, thus $u_{\epsilon}>0$ and $v_{\epsilon} \geq 0$ in $Q_{T}$ by Proposition 2.1. Multiplying by $u_{\epsilon}$ the first equation of (3), where $f(u, v)$ is replaced by $\rho f(u, v)$, integrating over $\Omega$ and using the Steklov averages $\left(u_{\epsilon}\right)_{h} \in H^{1}\left(Q_{T-\delta}\right), \delta, h>0$, (see $[15$, p.85]), we obtain

$$
\begin{aligned}
& \frac{1}{2} \frac{d}{d t} \log \int_{\Omega}\left(u_{\epsilon}\right)_{h}^{2} d x+\frac{\int_{\Omega}\left(m\left(u_{\epsilon}\right)_{h}^{m-1}+\epsilon\right)\left|\nabla\left(u_{\epsilon}\right)_{h}\right|^{2} d x}{\int_{\Omega}\left(u_{\epsilon}\right)_{h}^{2} d x} \\
& \leq \rho\left(\|a\|_{\infty}+\left\|K_{2}\right\|_{\infty} \int_{\Omega}\left(v_{\epsilon}\right)_{h}^{2}\left(\xi, t-\tau_{2}\right) d \xi\right) .
\end{aligned}
$$

Since $t \mapsto\|u(t)\|_{L^{2}(\Omega)}$ is continuous in $[0, T]$, there exist $t_{1}$ and $t_{2}$ in $[0, T]$ such that

$$
\int_{\Omega} u_{\epsilon}^{2}\left(x, t_{1}\right) d x=\min _{t \in[0, T]} \int_{\Omega} u_{\epsilon}^{2}(x, t) d x
$$

and

$$
\int_{\Omega} u_{\epsilon}^{2}\left(x, t_{2}\right) d x=\max _{t \in[0, T]} \int_{\Omega} u_{\epsilon}^{2}(x, t) d x .
$$

Integrating (13) between $t_{1}$ and $t_{2}$ and passing to the limit as $h \rightarrow 0$ we obtain

$$
\frac{1}{2}\left[\log \max _{t \in[0, T]} \int_{\Omega} u_{\epsilon}^{2}(x, t) d x-\log \min _{t \in[0, T]} \int_{\Omega} u_{\epsilon}^{2}(x, t) d x\right] \leq T\|a\|_{\infty}+\left\|K_{2}\right\|_{\infty} C_{2},
$$

or, equivalently,

$$
\max _{t \in[0, T]} \int_{\Omega} u_{\epsilon}^{2}(x, t) d x \leq C \min _{t \in[0, T]} \int_{\Omega} u_{\epsilon}^{2}(x, t) d x,
$$

where $C$ is independent of $\epsilon$ and $\rho$. Hence, there is a constant $\gamma>0$, independent of $\epsilon$ and $\rho$, such that

$$
\max _{t \in[0, T]} \int_{\Omega} u_{\epsilon}^{2}(x, t) d x \leq \gamma .
$$

Otherwise, inequality (14) would imply that the solutions $u_{\epsilon}$ are unbounded in $L^{2}\left(Q_{T}\right)$ as $\epsilon$ ranges in $(0,1 / 2)$ and $\rho$ in $(0,1]$, against our assumption (10). Of course, an analogous inequality holds for $v_{\epsilon}$. 
Now, we have

$$
\begin{aligned}
\frac{\partial u_{\epsilon}}{\partial t}-\Delta\left(u_{\epsilon}^{m}+\epsilon u_{\epsilon}\right) & \leq\left(\|a\|_{\infty}+\left\|K_{2}\right\|_{\infty} \max _{t \in[0, T]} \int_{\Omega} v_{\epsilon}^{2}(x, t) d x\right) u_{\epsilon} \\
& \leq\left(\|a\|_{\infty}+\left\|K_{2}\right\|_{\infty} \gamma\right) u_{\epsilon} .
\end{aligned}
$$

By [22, Lemma 2] we conclude that $\left\|u_{\epsilon}\right\|_{\infty} \leq R_{1}$ for some $R_{1}>0$ independent of $\rho$ and $\epsilon$. Analogously, $\left\|v_{\epsilon}\right\|_{\infty} \leq R_{2}$ for some constant $R_{2}>0$. Therefore it is enough to choose $R>\max \left\{R_{1}, R_{2}\right\}$.

The homotopy invariance property of the Leray-Schauder degree implies that

$$
\begin{aligned}
& \operatorname{deg}\left((u, v)-T_{\epsilon}\left(1, u^{+}, v^{+}\right), B_{R}, 0\right) \\
& \quad=\operatorname{deg}\left((u, v)-G_{\epsilon}\left(1, \rho f\left(u^{+}, v^{+}\right), \rho g\left(u^{+}, v^{+}\right)\right), B_{R}, 0\right)
\end{aligned}
$$

for any $\rho \in[0,1]$. If we take $\rho=0$, using the fact that $G_{\epsilon}$ at $\rho=0$ is the zero map, it results

$$
\operatorname{deg}\left((u, v)-T_{\epsilon}\left(1, u^{+}, v^{+}\right), B_{R}, 0\right)=\operatorname{deg}\left((u, v), B_{R}, 0\right)=1 .
$$

Now, let us assume that $\eta\left(C_{1}, C_{2}\right)>0$ and observe that this ensures that $(6)$ holds and that there are $R>r>0$, independent of $\epsilon$, such that

$$
\operatorname{deg}\left((u, v)-G_{\epsilon}\left(1, f\left(u^{+}, v^{+}\right), g\left(u^{+}, v^{+}\right)\right), B_{R} \backslash \bar{B}_{r}, 0\right)=1
$$

for any $\epsilon \in(0,1 / 2)$, by Proposition 2.2 and the excision property of the topological degree.

Let us fix any $\epsilon \in(0,1 / 2)$. There is $\sigma_{0}=\sigma_{0}(\epsilon) \in(0,1)$ such that still

$$
\operatorname{deg}\left((u, v)-G_{\epsilon}\left(\sigma, f\left(u^{+}, v^{+}\right)+1-\sigma, g\left(u^{+}, v^{+}\right)+1-\sigma\right), B_{R} \backslash \bar{B}_{r}, 0\right)=1
$$

for all $\sigma \in\left[\sigma_{0}, 1\right]$, by the continuity of Leray-Schauder degree. This implies that the set of solution triples $(\sigma, u, v) \in[0,1] \times\left(B_{R} \backslash \bar{B}_{r}\right)$ such that

$$
(u, v)=G_{\epsilon}\left(\sigma, f\left(u^{+}, v^{+}\right)+1-\sigma, g\left(u^{+}, v^{+}\right)+1-\sigma\right)
$$

contains a continuum $\mathcal{S}_{\epsilon}$ with the property that

$$
\mathcal{S}_{\epsilon} \cap\left[\{\sigma\} \times\left(B_{R} \backslash \bar{B}_{r}\right)\right] \neq \emptyset \quad \text { for all } \sigma \in\left[\sigma_{0}, 1\right] .
$$

Now, all the pairs $(u, v)$ such that $(1, u, v) \in \mathcal{S}_{\epsilon}$ are $T$-periodic solutions of (3) with $(u, v) \neq(0,0)$ and, hence, satisfy $(10)$. Since the $L^{2}$-norm is continuous with respect to the $L^{\infty}$-norm and $\mathcal{S}_{\epsilon}$ is a continuum, for every $\nu>0$ there is $\sigma_{\nu} \in\left[\sigma_{0}, 1\right)$ such that

$$
\|u\|_{2}^{2} \leq C_{1}+\nu \quad \text { and } \quad\|v\|_{2}^{2} \leq C_{2}+\nu
$$

for all $(u, v)$ with $(\sigma, u, v) \in \mathcal{S}_{\epsilon}$ and $\sigma \in\left[\sigma_{\nu}, 1\right]$. Observe that, if $(\sigma, u, v) \in \mathcal{S}_{\epsilon}$ for $\sigma<1$, then $u$ and $v$ are positive solutions of (16). Moreover, if $\nu$ is sufficiently small, then we still have $\eta\left(C_{1}+\nu, C_{2}+\nu\right)>0$. 
Now we can prove that, if $\nu$ is sufficiently small, then

$$
\begin{aligned}
\|u\|_{\infty},\|v\|_{\infty} \geq \lambda_{\nu}:=\min \{ & {\left[\frac{1}{2 m}\right]^{\frac{1}{m-1}},\left[\frac{T \eta\left(C_{1}+\nu, C_{2}+\nu\right)}{\left\|K_{1}\right\|_{1}}\right]^{\frac{1}{2}}, } \\
& {\left.\left[\frac{T \eta\left(C_{1}+\nu, C_{2}+\nu\right)}{\left\|K_{4}\right\|_{1}}\right]^{\frac{1}{2}}\right\} }
\end{aligned}
$$

for all $u, v$ such that $(\sigma, u, v) \in \mathcal{S}_{\epsilon}$ and $\sigma \in\left[\sigma_{\nu}, 1\right)$. Indeed, let $(u, v)$ be a solution of (16). Arguing by contradiction, assume that $\|u\|_{\infty}<\lambda_{\nu}$ and proceeding as in the proof of Proposition 2.2 (see (9) and recall that $u>0$ since $(u, v)$ solves (16) with $\sigma<1$ ) we obtain the inequality

$$
\iint_{Q_{T}} \phi_{1}^{2}(x) a(x, t) d x d t-\mu_{1} T<\left\|K_{1}\right\|_{1} \lambda_{\nu}^{2}+\underline{k}_{2}\left(C_{2}+\nu\right)
$$

Thus, the definition of $\eta$ implies that

$$
T \eta\left(C_{1}+\nu, C_{2}+\nu\right) \leq \iint_{Q_{T}} \phi_{1}^{2}(x) a(x, t) d x d t-\mu_{1} T-\underline{k}_{2}\left(C_{2}+\nu\right)<\left\|K_{1}\right\|_{1} \lambda_{\nu}^{2},
$$

which is a contradiction with the definition of $\lambda_{\nu}$. The same argument shows that $\|v\|_{\infty} \geq \lambda_{\nu}$.

Now, if we let $\sigma \rightarrow 1$ and $\nu \rightarrow 0$, then we obtain that (3) has at least a solution $\left(u_{\epsilon}, v_{\epsilon}\right)$ such that $\left\|u_{\epsilon}\right\|_{\infty},\left\|v_{\epsilon}\right\|_{\infty} \geq \lambda_{0}$, since $\mathcal{S}_{\epsilon}$ is a continuum and $\lambda_{\nu} \rightarrow \lambda_{0}$ as $\nu \rightarrow 0$.

Finally, we show that a solution $(u, v)$ of (1) with non-trivial $u, v \geq 0$ is obtained as a limit of $\left(u_{\epsilon}, v_{\epsilon}\right)$ as $\epsilon \rightarrow 0$ since $\lambda_{0}$ is independent of $\epsilon$. Indeed, from (15) we have

$$
\frac{\partial u_{\epsilon}}{\partial t}-\Delta\left(u_{\epsilon}^{m}+\epsilon u_{\epsilon}\right) \leq C u_{\epsilon}
$$

where $C$ is a positive constant independent of $\epsilon$. Multiplying (18) by $u_{\epsilon}^{m}$, integrating over $Q_{T}$ and passing to the limit in the Steklov averages $\left(u_{\epsilon}\right)_{h}$, one has

$$
\iint_{Q_{T}}\left|\nabla u_{\epsilon}^{m}\right|^{2} d x d t \leq \iint_{Q_{T}} \nabla\left(u_{\epsilon}^{m}+\epsilon u_{\epsilon}\right) \nabla u_{\epsilon}^{m} d x d t \leq C \iint_{Q_{T}} u_{\epsilon}^{m+1} d x d t \leq M,
$$

by the $T$-periodicity of $u_{\epsilon}$ and its boundedness in $L^{\infty}\left(Q_{T}\right)$, where $M$ is positive and independent of $\epsilon$. An analogous estimate holds for $v_{\epsilon}$.

Since $u_{\epsilon}, v_{\epsilon}$ are Hölder continuous in $\bar{Q}_{T}$, bounded in $C\left(\bar{Q}_{T}\right)$ uniformly in $\epsilon>0$ and the structure conditions of [19] are satisfied for the equations of system (1), whenever $\epsilon \in(0,1 / 2)$, Theorem 1.2 of [19] applies to conclude that the following inequality

$$
\left.\mid u_{\epsilon}\left(x_{1}, t_{1}\right)-u_{\epsilon}\left(x_{2}, t_{2}\right)\right) \mid \leq \Gamma\left(\left|x_{1}-x_{2}\right|^{\beta}+\left|t_{1}-t_{2}\right|^{\frac{\beta}{2}}\right)
$$


holds for any $\left(x_{1}, t_{1}\right),\left(x_{2}, t_{2}\right) \in \bar{Q}_{T}$, where the constants $\Gamma, \beta \in(0,1)$ are independent of $\left\|u_{\epsilon}\right\|_{\infty}$. The same inequality holds for $v_{\epsilon}$. Therefore, by the Ascoli-Arzelà Theorem, a subsequence of $\left(u_{\epsilon}, v_{\epsilon}\right)$ converges uniformly in $\bar{Q}_{T}$ to a pair $(u, v)$ satisfying

$$
\lambda_{0} \leq\|u\|_{\infty},\|v\|_{\infty} \leq R
$$

Moreover, by (19), $u_{\epsilon}^{m}, v_{\epsilon}^{m}$ are uniformly bounded in $L^{2}\left((0, T) ; H_{0}^{1}(\Omega)\right)$. Thus, some subsequences of $\nabla u_{\epsilon}^{m}$ and $\nabla v_{\epsilon}^{m}$ weakly converge in $L^{2}\left(Q_{T}\right)$. Hence $u^{m}, v^{m} \in$ $L^{2}\left((0, T) ; H_{0}^{1}(\Omega)\right)$, and by the convergence of $u_{\epsilon}$ and $v_{\epsilon}$ to $u$ and $v$ we obtain that $(u, v)$ is a weak solution of $(1)$.

Remark 2.2. Proposition 2.2, with assumption (6), does not guarantee that both components of a non-trivial solution $\left(u_{\epsilon}, v_{\epsilon}\right)$ of the regularized problem (3) are positive and, in fact, the proof of such a positivity is one of the main issues we had to handle in the proof of the preceding theorem with the help of the stronger assumption $\eta\left(C_{1}, C_{2}\right)>0$. However, if the cooperative case $K_{2}(x, t), K_{3}(x, t) \geq$ 0, i.e., $\underline{k}_{2}=\underline{k}_{3}=0$, is considered, then we have that

$\eta\left(C_{1}, C_{2}\right)=\min \left\{\frac{1}{T} \iint_{Q_{T}} \phi_{1}^{2}(x) a(x, t) d x d t-\mu_{1}, \frac{1}{T} \iint_{Q_{T}} \phi_{1}^{2}(x) b(x, t) d x d t-\mu_{1}\right\}=\eta_{0}$

and the condition $\eta_{0}>0$ is equivalent to (6). In particular, coexistence in the cooperative case follows from $\eta_{0}>0$ and a priori bounds on $\left(u_{\epsilon}, v_{\epsilon}\right)$, even if the constants $C_{1}, C_{2}$ are not explicitly known.

Remark 2.3. The assumption $\eta\left(C_{1}, C_{2}\right)>0$ is used to show (17) and, therefore, grants the non-triviality of both the components of the non-negative $T$ periodic solution $(u, v)$ that is given by Theorem 2.1. From a biological point of view, this hypothesis requires that the growth rates $a, b$ of the species are sufficiently large with respect to the terms that model the competition between them. In other words, it reasonably states that the competitive interaction between the two species should not prevail the growth capacity of the species if extinction has to be avoided.

However, when we proved the lower bounds (17), we used the estimate (9) for the first equation of the system in order to show that $\|u\|_{\infty}$ was not smaller than $\lambda_{\nu}$ and, implicitly, we used for $\|v\|_{\infty}$ the analogous estimate that holds for the second equation. On the other hand, if we use the second equation for $\|u\|_{\infty}$ (and the first equation for $\|v\|_{\infty}$ ) we obtain a different choice for $\lambda_{\nu}$ and, in particular, for $\eta$. In fact, we can prove a version of Theorem 2.1 with

$$
\begin{aligned}
\zeta\left(C_{1}, C_{2}\right):=\min \left\{\frac{1}{T} \iint_{Q_{T}} \phi_{1}^{2}(x) a(x, t) d x d t-\mu_{1}-\frac{\bar{k}_{1} C_{1}}{T},\right. \\
\left.\frac{1}{T} \iint_{Q_{T}} \phi_{1}^{2}(x) b(x, t) d x d t-\mu_{1}-\frac{\bar{k}_{4} C_{2}}{T}\right\}>0
\end{aligned}
$$

in place of $\eta\left(C_{1}, C_{2}\right)>0$, where $0 \leq K_{i}(x, t) \leq \bar{k}_{i}$ a.e. in $Q_{T}$ for $i=1,4$. 
Specifically, it turns out that

$$
\lambda_{\nu}:=\min \left\{\left[\frac{1}{2 m}\right]^{\frac{1}{m-1}},\left[\frac{T \zeta\left(C_{1}+\nu, C_{2}+\nu\right)}{\left\|K_{2}\right\|_{1}}\right]^{\frac{1}{2}},\left[\frac{T \zeta\left(C_{1}+\nu, C_{2}+\nu\right)}{\left\|K_{3}\right\|_{1}}\right]^{\frac{1}{2}}\right\}
$$

in (17). We observe that also the assumption $\zeta\left(C_{1}, C_{2}\right)>0$ has a biological meaning: it requires that the the competition inside each species does not prevail on the growth rate of the species itself.

The feasibility of the two conditions (12) and (20) depends on the constants $C_{1}, C_{2}$. As we will see in Section 3 there are cases, namely Theorems 3.1 and 3.3, in which one of them is never satisfied (see the next Remark 3.1).

\section{A priori bounds in $L^{2}\left(Q_{T}\right)$}

We apply Theorem 2.1 by looking for explicit a priori bounds in $L^{2}\left(Q_{T}\right)$ for the solutions of the approximating problems (3) in different situations. We consider two main different cases. In the first one, which we call the "coercive case", we assume that $K_{i}(x, t) \geq \underline{k}_{i}>0$ a.e. in $Q_{T}$ for $i=1,4$. In the second one, the "non-coercive case", we allow the non-negative functions $K_{1}, K_{4}$ to vanish on sets with positive measure. We distinguish also between cooperative and competitive situations by imposing sign conditions on $K_{2}, K_{3}$ and having in mind the biological interpretation of model (1).

\subsection{The coercive case}

Theorem 3.1. Assume $m>1$ and that

1. there are constants $\underline{k}_{i}>0, i=1,4$, and $\underline{k}_{i}, \bar{k}_{i} \geq 0, i=2,3$, such that $\underline{k}_{1} \underline{k}_{4}>\bar{k}_{2} \bar{k}_{3}$ and

$$
K_{i}(x, t) \geq \underline{k}_{i} \text { for } i=1,4 \quad \text { and } \quad-\underline{k}_{i} \leq K_{i}(x, t) \leq \bar{k}_{i} \text { for } i=2,3,
$$

for a.a. $(x, t) \in Q_{T}$;

2. condition (12) of Theorem 2.1, that is $\eta\left(C_{1}, C_{2}\right)>0$, is satisfied with

$$
C_{1}=\frac{T \underline{k}_{4}}{\underline{k}_{1} \underline{k}_{4}-\bar{k}_{2} \bar{k}_{3}}\left(\|a\|_{\infty}+\frac{\bar{k}_{2}}{\underline{k}_{4}}\|b\|_{\infty}\right)
$$

and

$$
C_{2}=\frac{T \underline{k}_{1}}{\underline{k}_{1} \underline{k}_{4}-\bar{k}_{2} \bar{k}_{3}}\left(\|b\|_{\infty}+\frac{\bar{k}_{3}}{\underline{k}_{1}}\|a\|_{\infty}\right) .
$$

Then problem (1) has a non-negative T-periodic solution $(u, v)$ with non-trivial $u, v$.

Proof. We just need to show that $\left\|u_{\epsilon}\right\|_{2}^{2} \leq C_{1}$ and $\left\|v_{\epsilon}\right\|_{2}^{2} \leq C_{2}$ for any solution $\left(u_{\epsilon}, v_{\epsilon}\right)$ of (11). Then, assume $u_{\epsilon} \neq 0$, thus $u_{\epsilon}>0$ and $v_{\epsilon} \geq 0$ in $Q_{T}$ by 
Proposition 2.1. Integrating the equation (13) over $[0, T]$, and passing to the limit as $h \rightarrow 0$, by the $T$-periodicity of $u_{\epsilon}$, we have that

$$
\begin{aligned}
\int_{0}^{T} \frac{\epsilon \int_{\Omega}\left|\nabla u_{\epsilon}\right|^{2} d x}{\int_{\Omega} u_{\epsilon}^{2} d x} d t & \leq \int_{0}^{T} \frac{\int_{\Omega}\left(m u_{\epsilon}^{m-1}+\epsilon\right)\left|\nabla u_{\epsilon}\right|^{2} d x}{\int_{\Omega} u_{\epsilon}^{2} d x} d t \\
& \leq T\|a\|_{\infty}-\underline{k}_{1}\left\|u_{\epsilon}\right\|_{2}^{2}+\bar{k}_{2}\left\|v_{\epsilon}\right\|_{2}^{2}
\end{aligned}
$$

and, by Poincaré's inequality:

$$
\epsilon \mu_{1} T \leq T\|a\|_{\infty}-\underline{k}_{1}\left\|u_{\epsilon}\right\|_{2}^{2}+\bar{k}_{2}\left\|v_{\epsilon}\right\|_{2}^{2}
$$

The same procedure, when it is applied to the second equation of (3), leads to

$$
\epsilon \mu_{1} T \leq T\|b\|_{\infty}-\underline{k}_{4}\left\|v_{\epsilon}\right\|_{2}^{2}+\bar{k}_{3}\left\|u_{\epsilon}\right\|_{2}^{2}
$$

Hence from (21) and (22) we have

$$
\begin{aligned}
\left\|u_{\epsilon}\right\|_{2}^{2} & \leq \frac{T\|a\|_{\infty}+\bar{k}_{2}\left\|v_{\epsilon}\right\|_{2}^{2}-\epsilon \mu_{1} T}{\underline{k}_{1}} \\
\left\|v_{\epsilon}\right\|_{2}^{2} & \leq \frac{T\|b\|_{\infty}+\bar{k}_{3}\left\|u_{\epsilon}\right\|_{2}^{2}-\epsilon \mu_{1} T}{\underline{k}_{4}}
\end{aligned}
$$

These two inequalities imply that

$$
\begin{aligned}
& \left(1-\frac{\bar{k}_{2} \bar{k}_{3}}{\underline{k}_{1} \underline{k}_{4}}\right)\left\|u_{\epsilon}\right\|_{2}^{2}<\frac{T}{\underline{k}_{1}}\left(\|a\|_{\infty}+\frac{\bar{k}_{2}}{\underline{k}_{4}}\|b\|_{\infty}\right) \\
& \left(1-\frac{\bar{k}_{2} \bar{k}_{3}}{\underline{k}_{1} \underline{k}_{4}}\right)\left\|v_{\epsilon}\right\|_{2}^{2}<\frac{T}{\underline{k}_{4}}\left(\|b\|_{\infty}+\frac{\bar{k}_{3}}{\underline{k}_{1}}\|a\|_{\infty}\right)
\end{aligned}
$$

for any $\epsilon \in(0,1 / 2)$ and the desired bounds follow since $\bar{k}_{2} \bar{k}_{3}<\underline{k}_{1} \underline{k}_{4}$.

As immediate consequences of the previous result we obtain the following corollaries for the cooperative and the competitive cases.

Corollary 3.1. Assume $m>1$ and that $K_{i}(x, t) \geq \underline{k}_{i}>0$ for a.a. $(x, t) \in Q_{T}$ and $i=1,4$. If the system is cooperative, i.e. $\underline{k}_{2}, \underline{k}_{3}=0$, and (6) holds, then problem (1) has a non-negative T-periodic solution (u,v) with non-trivial $u, v$.

Corollary 3.2. Assume $m>1$ and that $K_{i}(x, t) \geqq \underline{k}_{i}>0$ for a.a. $(x, t) \in Q_{T}$ and $i=1,4$. If the system is competitive, i.e. $\bar{k}_{2}, \bar{k}_{3}=0$, then problem (1) has a non-negative $T$-periodic solution $(u, v)$, with non-trivial $u, v$, provided that

$$
\begin{aligned}
\min \{ & \frac{1}{T} \iint_{Q_{T}} \phi_{1}^{2}(x) a(x, t) d x d t-\mu_{1}-\frac{\underline{k}_{2}}{\underline{k}_{4}}\|b\|_{\infty} ; \\
& \left.\frac{1}{T} \iint_{Q_{T}} \phi_{1}^{2}(x) b(x, t) d x d t-\mu_{1}-\frac{\underline{k}_{3}}{\underline{k}_{1}}\|a\|_{\infty}\right\}>0 .
\end{aligned}
$$


We observe that the condition $\bar{k}_{2} \bar{k}_{3}<\underline{k}_{1} \underline{k}_{4}$ of Theorem 3.1 is crucial to establish the a priori $L^{2}$-bounds on the solution pairs $\left(u_{\epsilon}, v_{\epsilon}\right)$ of (3). Roughly speaking this condition guarantees that the terms in the equations that contribute to the growth of the respective species do not prevail on the whole on those limiting the growth.

On the other hand, when the strict positivity of the functions $K_{1}$ and $K_{4}$ is relaxed, obtaining the needed a priori bounds becomes more difficult (at least with our approach). In fact, we are able to obtain simple a priori bounds in the non-coercive case when some sign condition is imposed on the functions $K_{2}$ and $K_{3}$ (weak and strong competition, see Subsections 3.2 and 3.3), but we have to impose the technical restriction $m>3$ to obtain a result like Theorem 3.1 with no sign condition on the functions $K_{2}$ and $K_{3}$ (and with rather complicated constants $C_{1}, C_{2}$, see Subsection 3.4).

\subsection{The non-coercive case: weak competition and $\mathbf{m}>\mathbf{1}$}

Theorem 3.2. Assume $m>1$ and that

1. $K_{i}(x, t) \geq 0, i=1,4$ and $-\underline{k}_{i} \leq K_{i}(x, t) \leq 0, i=2,3$ for a. $a .(x, t) \in Q_{T}$ and for some non-negative constants $\underline{k}_{i}, i=2,3$;

2. condition (12) of Theorem 2.1, that is $\eta\left(C_{1}, C_{2}\right)>0$, is satisfied with

$$
C_{1}=T|\Omega|\left(\frac{(m+1)^{2}}{4 m \mu_{1}}\|a\|_{\infty}\right)^{\frac{2}{m-1}}
$$

and

$$
C_{2}=T|\Omega|\left(\frac{(m+1)^{2}}{4 m \mu_{1}}\|b\|_{\infty}\right)^{\frac{2}{m-1}} .
$$

Then problem (1) has a T-periodic non-negative solution $(u, v)$ with non-trivial $u, v$.

ProOF. We begin by finding the bound for the solutions $u_{\epsilon}$ of the first equation of (11). Since, by Hölder's inequality,

$$
\int_{\Omega} u_{\epsilon}^{2} d x \leq|\Omega|^{\frac{m-1}{m+1}}\left(\int_{\Omega} u_{\epsilon}^{m+1} d x\right)^{\frac{2}{m+1}},
$$

and, by Poincaré's inequality,

$$
\begin{aligned}
\int_{\Omega}\left|u_{\epsilon}^{\frac{m+1}{2}}\right|^{2} d x & \leq \frac{1}{\mu_{1}} \int_{\Omega}\left|\nabla u_{\epsilon}^{\frac{m+1}{2}}\right|^{2} d x=\frac{(m+1)^{2}}{4 \mu_{1}} \int_{\Omega} u_{\epsilon}^{m-1}\left|\nabla u_{\epsilon}\right|^{2} d x \\
& \leq \frac{(m+1)^{2}}{4 m \mu_{1}} \int_{\Omega}\left(m u_{\epsilon}^{m-1}+\epsilon\right)\left|\nabla u_{\epsilon}\right|^{2} d x
\end{aligned}
$$

we obtain

$$
\int_{\Omega} u_{\epsilon}^{2} d x \leq|\Omega|^{\frac{m-1}{m+1}}\left(\frac{(m+1)^{2}}{4 m \mu_{1}}\right)^{\frac{2}{m+1}}\left(\int_{\Omega}\left(m u_{\epsilon}^{m-1}+\epsilon\right)\left|\nabla u_{\epsilon}\right|^{2} d x\right)^{\frac{2}{m+1}} .
$$


Integrating over $[0, T]$ it results

$$
\begin{aligned}
\left\|u_{\epsilon}\right\|_{2}^{2} & \leq|\Omega|^{\frac{m-1}{m+1}}\left(\frac{(m+1)^{2}}{4 m \mu_{1}}\right)^{\frac{2}{m+1}} \int_{0}^{T}\left(\int_{\Omega}\left(m u_{\epsilon}^{m-1}+\epsilon\right)\left|\nabla u_{\epsilon}\right|^{2} d x\right)^{\frac{2}{m+1}} d t \\
& \leq|\Omega|^{\frac{m-1}{m+1}}\left(\frac{(m+1)^{2}}{4 m \mu_{1}}\right)^{\frac{2}{m+1}} T^{\frac{m-1}{m+1}}\left(\iint_{Q_{T}}\left(m u_{\epsilon}^{m-1}+\epsilon\right)\left|\nabla u_{\epsilon}\right|^{2} d x d t\right)^{\frac{2}{m+1}}
\end{aligned}
$$

by Hölder's inequality with $p=\frac{m+1}{2}$. Multiplying the first equation of (11) by $u_{\epsilon}$, integrating in $Q_{T}$ and passing to the limit in the Steklov averages $\left(u_{\epsilon}\right)_{h}$, we obtain

$$
\iint_{Q_{T}}\left(m u_{\epsilon}^{m-1}+\epsilon\right)\left|\nabla u_{\epsilon}\right|^{2} d x d t \leq\|a\|_{\infty}\left\|u_{\epsilon}\right\|_{2}^{2}
$$

by the $T$-periodicity of $u_{\epsilon}$ and the non-positivity of the function $K_{2}$, and, therefore,

$$
\left\|u_{\epsilon}\right\|_{2}^{2} \leq T|\Omega|\left(\frac{(m+1)^{2}}{4 m \mu_{1}}\|a\|_{\infty}\right)^{\frac{2}{m-1}}=C_{1} .
$$

In an analogous way we obtain that $\left\|v_{\epsilon}\right\|_{2}^{2} \leq C_{2}$, if $v_{\epsilon}$ is a solution of the second equation of (11).

The arguments of the proof of Theorem 3.2 can be easily adapted to show the following result for the case of a single equation with a non-coercive local term.

Corollary 3.3. Assume $m>1$ and that the function $K$ is non-negative. If

$$
\frac{1}{T} \iint_{Q_{T}} \phi_{1}^{2}(x) a(x, t) d x d t-\mu_{1}>0,
$$

then the problem

$$
\left\{\begin{array}{l}
\frac{\partial u}{\partial t}-\Delta u^{m}=\left(a(x, t)-\int_{\Omega} K(\xi, t) u^{2}(\xi, t-\tau) d \xi\right) u \\
\left.u(\cdot, t)\right|_{\partial \Omega}=0, \quad \text { for a.a. } \quad t \in(0, T) \\
u(\cdot, 0)=u(\cdot, T),
\end{array}\right.
$$

has a non-trivial T-periodic solution $u \geq 0$.

3.3. The non-coercive case: strong competition and $\mathbf{m}>\mathbf{1}$

Theorem 3.3. Assume $m>1$ and that

1. $K_{i}(x, t) \geq 0, i=1,4$ and $-\underline{k}_{i} \leq K_{i}(x, t) \leq-\bar{k}_{i}<0, i=2,3$ for a.a. $(x, t) \in Q_{T}$ and for some positive constants $\underline{k}_{i}, \bar{k}_{i}, i=2,3$;

2. condition $(20)$, i.e. $\zeta\left(C_{1}, C_{2}\right)>0$, is satisfied with

$$
\begin{aligned}
& C_{1}=T \max \left\{|\Omega|\left(\frac{(m+1)^{2}}{4 m \mu_{1}}\|a\|_{\infty}\right)^{\frac{2}{m-1}}, \frac{\|b\|_{\infty}}{\bar{k}_{3}}\right\} \\
& C_{2}=T \max \left\{|\Omega|\left(\frac{(m+1)^{2}}{4 m \mu_{1}}\|b\|_{\infty}\right)^{\frac{2}{m-1}}, \frac{\|a\|_{\infty}}{\bar{k}_{2}}\right\} .
\end{aligned}
$$


Then problem (1) has a non-negative T-periodic solution $(u, v)$ with non-trivial $u, v$.

PROOF. If $\left(u_{\epsilon}, v_{\epsilon}\right)$ is a solution of $(11)$ with $u_{\epsilon} \equiv 0$, or respectively $v_{\epsilon} \equiv 0$, one can argue as in the proof of Theorem 3.2 to obtain that

$$
\left\|v_{\epsilon}\right\|_{2}^{2} \leq T|\Omega|\left(\frac{(m+1)^{2}}{4 m \mu_{1}}\|b\|_{\infty}\right)^{\frac{2}{m-1}}
$$

or respectively,

$$
\left\|u_{\epsilon}\right\|_{2}^{2} \leq T|\Omega|\left(\frac{(m+1)^{2}}{4 m \mu_{1}}\|a\|_{\infty}\right)^{\frac{2}{m-1}}
$$

If $u_{\epsilon} \neq 0$, then $u_{\epsilon}>0$ and $v_{\epsilon} \geq 0$ in $Q_{T}$ by Proposition 2.1. Moreover $u_{\epsilon} \in$ $C\left(\bar{Q}_{T}\right)$ and, hence, there exists $t_{1} \in[0, T]$ such that

$$
\int_{\Omega} u_{\epsilon}^{2}\left(x, t_{1}\right) d x=\min _{t \in[0, T]} \int_{\Omega} u_{\epsilon}^{2}(x, t) d x
$$

Multiplying the first equation of (11) by $u_{\epsilon}$, integrating over $\Omega$ and using the Steklov averages $\left(u_{\epsilon}\right)_{h}$ we obtain

$$
\frac{1}{2} \frac{d}{d t} \int_{\Omega}\left(u_{\epsilon}\right)_{h}^{2} d x \leq\left(\|a\|_{\infty}-\bar{k}_{2} \int_{\Omega}\left(v_{\epsilon}\right)_{h}^{2}\left(\xi, t-\tau_{2}\right) d \xi\right) \int_{\Omega}\left(u_{\epsilon}\right)_{h}^{2} d x
$$

Hence, we have

$$
\frac{d}{d t}\left[\exp \left\{2 \int_{t_{1}}^{t}\left(\bar{k}_{2} \int_{\Omega}\left(v_{\epsilon}\right)_{h}^{2}\left(\xi, s-\tau_{2}\right) d \xi-\|a\|_{\infty}\right) d s\right\} \int_{\Omega}\left(u_{\epsilon}\right)_{h}^{2}(x, t) d x\right] \leq 0
$$

for $t \geq t_{1}$, which implies that

$\exp \left\{2 \int_{t_{1}}^{t}\left(\bar{k}_{2} \int_{\Omega}\left(v_{\epsilon}\right)_{h}^{2}\left(\xi, s-\tau_{2}\right) d \xi-\|a\|_{\infty}\right) d s\right\} \int_{\Omega}\left(u_{\epsilon}\right)_{h}^{2}(x, t) d x \leq \int_{\Omega}\left(u_{\epsilon}\right)_{h}^{2}\left(x, t_{1}\right) d x$ for $t \geq t_{1}$, and, passing to the limit as $h \rightarrow 0$ and taking $t=t_{1}+T$,

$$
\begin{aligned}
& \exp \left\{2 \bar{k}_{2} \int_{t_{1}}^{t_{1}+T} \int_{\Omega} v_{\epsilon}^{2}\left(\xi, t-\tau_{2}\right) d \xi d t-2 T\|a\|_{\infty}\right\} \int_{\Omega} u_{\epsilon}^{2}\left(x, t_{1}+T\right) d x \\
& \leq \int_{\Omega} u_{\epsilon}^{2}\left(x, t_{1}\right) d x \leq \int_{\Omega} u_{\epsilon}^{2}\left(x, t_{1}+T\right) d x .
\end{aligned}
$$

Therefore we have that

$$
\iint_{Q_{T}} v_{\epsilon}^{2}(x, t) d x d t=\int_{t_{1}}^{t_{1}+T} \int_{\Omega} v_{\epsilon}^{2}\left(\xi, t-\tau_{2}\right) d \xi d t \leq \frac{T\|a\|_{\infty}}{\bar{k}_{2}}
$$

by the $T$-periodicity of $v_{\epsilon}$.

If $v_{\epsilon} \neq 0$, then we can prove that $\left\|u_{\epsilon}\right\|_{2}^{2} \leq T\|b\|_{\infty} / \bar{k}_{3}$ in a similar way. Finally, Remark 2.3 allows to apply Theorem 2.1 with (12) replaced by (20).

Observe that the conditions $\bar{k}_{2}>0$ and $\bar{k}_{3}>0$ are essential to establish the a priori bounds of the last theorem. 


\subsection{The non-coercive case: $\mathbf{m}>\mathbf{3}$}

If $m>3$, we are able to find explicit bounds (although complicated) without any assumption on the sign of the functions $K_{2}, K_{3}$, as shown in the next result.

Theorem 3.4. Assume $m>3$ and that

1. $K_{i}(x, t) \geq 0, i=1,4$ and $K_{i}(x, t) \leq \bar{k}_{i}, i=2,3$ for a.a. $(x, t) \in Q_{T}$ and for some positive constants $\bar{k}_{i}, i=2,3$;

2. condition (12) of Theorem 2.1, that is $\eta\left(C_{1}, C_{2}\right)>0$, is satisfied with

$$
\begin{aligned}
C_{1}= & T\left\{\frac{(m-1)^{2}}{(m-3)(m+1)}\left[\left(M\|a\|_{\infty}^{2}\right)^{\frac{2}{m-1}}+\left(M^{\frac{m+1}{m-1}} \bar{k}_{2}^{2}\|b\|_{\infty}^{\frac{4}{m-1}}\right)^{\frac{2}{m-1}}\right]+\right. \\
& \left.+\left(M \bar{k}_{2}^{\frac{2 m-2}{m+1}} \bar{k}_{3}^{\frac{4}{m+1}}\right)^{\frac{2}{m-3}}\right\}^{1 / 2}, \\
C_{2}= & T\left\{\frac{(m-1)^{2}}{(m-3)(m+1)}\left[\left(M\|b\|_{\infty}^{2}\right)^{\frac{2}{m-1}}+\left(M^{\frac{m+1}{m-1}} \bar{k}_{3}^{2}\|a\|_{\infty}^{\frac{4}{m-1}}\right)^{\frac{2}{m-1}}\right]+\right. \\
& \left.+\left(M \bar{k}_{3}^{\frac{2 m-2}{m+1}} \bar{k}_{2}^{\frac{4}{m+1}}\right)^{\frac{2}{m-3}}\right\}^{1 / 2}, \\
M= & \frac{(m+1)^{4}|\Omega|^{m-1}}{8 m^{2} \mu_{1}^{2}} .
\end{aligned}
$$

Then problem (1) has a non-negative T-periodic solution $(u, v)$ with non-trivial $u, v$.

Proof. Let $\left(u_{\epsilon}, v_{\epsilon}\right)$ be a solution of (11). We have

$$
\left(\int_{\Omega} u_{\epsilon}^{2} d x\right)^{\frac{m+1}{2}} \leq\left(\frac{M}{2}\right)^{1 / 2} \int_{\Omega}\left(m u_{\epsilon}^{m-1}+\epsilon\right)\left|\nabla u_{\epsilon}\right|^{2} d x
$$

by inequality (23). Multiplying the first equation of (11) by $u_{\epsilon}$, integrating in $Q_{T}$ and passing to the limit in the Steklov averages $\left(u_{\epsilon}\right)_{h}$, we obtain by the $T$-periodicity of $u_{\epsilon}$

$$
\iint_{Q_{T}}\left(m u_{\epsilon}^{m-1}+\epsilon\right)\left|\nabla u_{\epsilon}\right|^{2} d x \leq \int_{0}^{T}\left(\|a\|_{\infty}+\bar{k}_{2} \int_{\Omega} v_{\epsilon}^{2}\left(\xi, t-\tau_{2}\right) d \xi\right)\left(\int_{\Omega} u_{\epsilon}^{2} d x\right) d t .
$$

It follows that

$$
\begin{aligned}
\int_{0}^{T}\left(\int_{\Omega} u_{\epsilon}^{2}\right)^{\frac{m+1}{2}} d t & \leq\left[\frac{M}{2} \int_{0}^{T}\left(\|a\|_{\infty}+\bar{k}_{2} \int_{\Omega} v_{\epsilon}^{2}\left(\xi, t-\tau_{2}\right) d \xi\right)^{2} d t\right]^{\frac{1}{2}} . \\
\cdot & {\left[\int_{0}^{T}\left(\int_{\Omega} u_{\epsilon}^{2} d x\right)^{2} d t\right]^{\frac{1}{2}} }
\end{aligned}
$$


by (25), (26) and Hölder's inequality. On the other hand,

$$
\int_{0}^{T}\left(\int_{\Omega} u_{\epsilon}^{2} d x\right)^{2} d t \leq T^{\frac{m-3}{m+1}}\left[\int_{0}^{T}\left(\int_{\Omega} u_{\epsilon}^{2} d x\right)^{\frac{m+1}{2}} d t\right]^{\frac{4}{m+1}}
$$

by Hölder's inequality with $p=\frac{m+1}{4}$. Therefore, these last two inequalities imply

$$
\begin{aligned}
& \int_{0}^{T}\left(\int_{\Omega} u_{\epsilon}^{2} d x\right)^{2} d t \leq T^{\frac{m-3}{m-1}}\left(\frac{M}{2}\right)^{\frac{2}{m-1}}\left[\int_{0}^{T}\left(\|a\|_{\infty}+\bar{k}_{2} \int_{\Omega} v_{\epsilon}^{2} d x\right)^{2} d t\right]^{\frac{2}{m-1}} \\
& \leq T^{\frac{m-3}{m-1}}\left(\frac{M}{2}\right)^{\frac{2}{m-1}}\left[2 T\|a\|_{\infty}^{2}+2 \bar{k}_{2}^{2} \int_{0}^{T}\left(\int_{\Omega} v_{\epsilon}^{2} d x\right)^{2} d t\right]^{\frac{2}{m-1}} \\
& \leq T\left(M\|a\|_{\infty}^{2}\right)^{\frac{2}{m-1}}+T^{\frac{m-3}{m-1}}\left(M \bar{k}_{2}^{2}\right)^{\frac{2}{m-1}}\left[\int_{0}^{T}\left(\int_{\Omega} v_{\epsilon}^{2} d x\right)^{2} d t\right]^{\frac{2}{m-1}}
\end{aligned}
$$

thanks to the fact that $m>3$. In an analogous way we can show that

$$
\begin{aligned}
\int_{0}^{T}\left(\int_{\Omega} v_{\epsilon}^{2} d x\right)^{2} d t & \leq T\left(M\|b\|_{\infty}^{2}\right)^{\frac{2}{m-1}}+ \\
& +T^{\frac{m-3}{m-1}}\left(M \bar{k}_{3}^{2}\right)^{\frac{2}{m-1}}\left[\int_{0}^{T}\left(\int_{\Omega} u_{\epsilon}^{2} d x\right)^{2} d t\right]^{\frac{2}{m-1}}
\end{aligned}
$$

and, hence,

$$
\begin{aligned}
U & \leq T\left(M\|a\|_{\infty}^{2}\right)^{\frac{2}{m-1}}+ \\
& +T^{\frac{m-3}{m-1}}\left(M \bar{k}_{2}^{2}\right)^{\frac{2}{m-1}}\left[T\left(M\|b\|_{\infty}^{2}\right)^{\frac{2}{m-1}}+T^{\frac{m-3}{m-1}}\left(M \bar{k}_{3}^{2}\right)^{\frac{2}{m-1}} U^{\frac{2}{m-1}}\right]^{\frac{2}{m-1}} \\
& \leq T\left(M\|a\|_{\infty}^{2}\right)^{\frac{2}{m-1}}+T\left(M^{\frac{m+1}{m-1}} \bar{k}_{2}^{2}\|b\|_{\infty}^{\frac{4}{m-1}}\right)^{\frac{2}{m-1}}+ \\
& +\left(T^{\frac{(m-3)(m+1)}{2(m-1)}} M^{\frac{m+1}{m-1}} \bar{k}_{2}^{2} \bar{k}_{3}^{\frac{4}{m-1}}\right)^{\frac{2}{m-1}} U^{\frac{4}{(m-1)^{2}}}
\end{aligned}
$$

with $U=\int_{0}^{T}\left(\int_{\Omega} u_{\epsilon}^{2} d x\right)^{2} d t$. The last inequality has the form:

$$
U \leq \alpha+\beta U^{\frac{4}{(m-1)^{2}}},
$$

with $\alpha, \beta>0$. Since $m>3$ the function $f(U):=\alpha+\beta U^{\frac{4}{(m-1)^{2}}}$ is concave, and then

$$
f(U) \leq f\left(U_{0}\right)+f^{\prime}\left(U_{0}\right)\left(U-U_{0}\right),
$$


where $U_{0}:=\beta^{\frac{(m-1)^{2}}{(m-3)(m+1)}}$. This implies

$$
U \leq \frac{(m-1)^{2}}{(m-3)(m+1)} \alpha+\beta^{\frac{(m-1)^{2}}{(m-3)(m+1)}} .
$$

A final application of Hölder's inequality shows that $\left\|u_{\epsilon}\right\|_{2}^{2} \leq T^{1 / 2} U^{1 / 2}=C_{1}$. The argument for $v_{\epsilon}$ proceeds in a similar way.

In the cooperative case we can ignore the explicit value of the constants in (24) and obtain the following cleaner looking corollary thanks to Remark 2.2.

Corollary 3.4. Assume $m>3$ and that $K_{1}(x, t), K_{4}(x, t) \geq 0$ for a.a. $(x, t) \in$ $Q_{T}$. If the system is cooperative, i.e. $\underline{k}_{2}, \underline{k}_{3}=0$, and (6) holds, then problem (1) has a non-negative T-periodic solution $(u, v)$ with non-trivial $u, v$.

Remark 3.1. Observe that the a priori bounds $C_{1}, C_{2}$ in Theorem 3.1 do not allow to replace the condition (12) with (20), since

$$
\min \left\{\frac{\bar{k}_{1} \underline{k}_{4}}{\underline{k}_{1} \underline{k}_{4}-\bar{k}_{2} \bar{k}_{3}}, \frac{\underline{k}_{1} \bar{k}_{4}}{\underline{k}_{1} \underline{k}_{4}-\bar{k}_{2} \bar{k}_{3}}\right\} \geq 1,
$$

which implies that $\zeta\left(C_{1}, C_{2}\right) \leq-\mu_{1}$. Analogously, in Theorem 3.3 the condition (20) cannot be replaced by (12) since in this case we have that

$$
\min \left\{\frac{\underline{k}_{2}}{\bar{k}_{2}}, \frac{\underline{k}_{3}}{\bar{k}_{3}}\right\} \geq 1
$$

which implies that $\eta\left(C_{1}, C_{2}\right) \leq-\mu_{1}$. Finally, in the other cases, namely Theorems 3.2 and 3.4, the constants $C_{1}, C_{2}$ allow to employ indifferently any of the two conditions.

\section{An optimization problem}

In this section we associate to (1) a cost function $J$ defined by

$$
J(u, v, a, b):=\iint_{Q_{T}} F(x, t, u(x, t), v(x, t), a(x, t), b(x, t)) d x d t
$$

to be minimized on all the pairs $(u, v, a, b)$ which satisfy (1) with $u, v$ non-trivial, non-negative in $Q_{T}$ and $a, b$ fulfilling either condition (12) of Theorem 2.1 or condition (20) of Remark 2.3. Moreover, we assume that $F: Q_{T} \times \mathbb{R}^{4} \rightarrow \mathbb{R}$ is a Carathéodory function which is measurable in $(x, t)$ for any $(u, v, a, b)$ and continuous with respect to $(u, v, a, b)$ for a.a. $(x, t) \in Q_{T}$. As a possible choice of $F$ we can consider $F(x, t, u, v, a, b):=C(x, t, a, b)-G(x, t, u, v, a, b)$, where $C$ represents the cost of controlling the natural growth rates to the values $a$ and $b$, and $G$ represents the benefit due to the presence of the populations of densities $u, v$ and growth rates $a, b$. We consider the above stated optimization 
problem both in the coercive and noncoercive case for cooperative and competitive systems, which are, in our opinion, the most relevant cases. To this aim we introduce the following set of admissible controls

$$
U=\left\{(a, b) \in L^{\infty}\left(Q_{T}\right) \times L^{\infty}\left(Q_{T}\right): \mu_{1}+\delta \leq a(x, t), b(x, t) \leq c \text { a.e. in } Q_{T}\right\}
$$

for some fixed $\delta, c>0$. In the coercive case, Corollary 3.1 guarantees that if the system is cooperative then, for any $(a, b) \in U$ with any positive constants $\delta, c$ such that $\delta+\mu_{1}<c$, (1) has non-negative solution $(u, v)$ with non-trivial $u, v$. Furthermore, Corollary 3.2 ensures that the same holds if the system is competitive for $\delta, c>0$ such that $\delta+\mu_{1}<c$ and

$$
\frac{\delta}{c}>\max \left\{\frac{\underline{k}_{2}}{\underline{k}_{4}}, \frac{\underline{k}_{3}}{\underline{k}_{1}}\right\}
$$

In the non-coercive case for cooperative systems, Corollary 3.4 allows the same conclusion of the coercive, cooperative case. While, if the system is weak competitive for $\delta, c>0$ such that

$$
\frac{\delta^{\frac{m-1}{2}}}{c}>\max \left\{\underline{k}_{2}^{\frac{m-1}{2}}, \underline{k}_{3}^{\frac{m-1}{2}}\right\}|\Omega|^{\frac{m-1}{2}} \frac{(m+1)^{2}}{4 m \mu_{1}}
$$

Theorem 3.2 applies for any $(a, b) \in U$. Finally, if the system is strongly competitive then Theorem 3.3 applies for any $(a, b) \in U$, where $\delta, c>0$ satisfy the conditions

$$
\frac{\delta^{\frac{m-1}{2}}}{c}>\max \left\{\bar{k}_{1}^{\frac{m-1}{2}}, \bar{k}_{4}^{\frac{m-1}{2}}\right\}|\Omega|^{\frac{m-1}{2}} \frac{(m+1)^{2}}{4 m \mu_{1}}
$$

and

$$
\frac{\delta}{c}>\max \left\{\frac{\bar{k}_{1}}{\bar{k}_{3}}, \frac{\bar{k}_{4}}{\bar{k}_{2}}\right\}
$$

To the control set $U$ we associate the solution set

$$
\begin{aligned}
S:=\{(u, v, a, b): & (u, v) \in C\left(\bar{Q}_{T}\right) \times C\left(\bar{Q}_{T}\right) \text { is a weak solution of }(1) \\
& \text { corresponding to }(a, b) \in U \text { such that } u, v \geq 0 \text { in } Q_{T}, \\
& \left.u, v \neq 0 \text { and } \lambda_{0}<\|u\|_{C\left(\bar{Q}_{T}\right)},\|v\|_{C\left(\bar{Q}_{T}\right)}<R\right\},
\end{aligned}
$$

where the constants $\lambda_{0}$ and $R$ are given by Theorem 2.1 .

In order to deal with the minimization of the cost functional $J$ on $S$ we give some preliminary definitions. Fixed $(a, b) \in U$, consider the function $\psi_{a, b}$ : $Q_{T} \times \mathbb{R}^{6} \rightarrow \mathbb{R}^{3}$ given by

$$
\begin{aligned}
\psi_{a, b}\left(x, t, \alpha, \beta, \alpha_{1}, \beta_{1}, \alpha_{2}, \beta_{2}\right):= & \left\{\left(a(x, t)-\alpha_{1}+\beta_{1}\right) \alpha,\left(b(x, t)+\alpha_{2}-\beta_{2}\right) \beta,\right. \\
& F(x, t, \alpha, \beta, a(x, t), b(x, t))\},
\end{aligned}
$$

and the multivalued map $\Psi: Q_{T} \times \mathbb{R}^{6} \multimap \mathbb{R}^{3}$ defined as follows:

$$
\begin{aligned}
\Psi\left(x, t, \alpha, \beta, \alpha_{1}, \beta_{1}, \alpha_{2}, \beta_{2}\right):= & \left\{\left(\gamma_{1}-\alpha_{1}+\beta_{1}\right) \alpha,\left(\gamma_{2}+\alpha_{2}-\beta_{2}\right) \beta,\right. \\
& \left.F\left(x, t, \alpha, \beta, \gamma_{1}, \gamma_{2}\right): \gamma_{1}, \gamma_{2} \in\left[\mu_{1}+\delta, c\right]\right\} .
\end{aligned}
$$


In what follows, abusing notations, we denote $X \times X \times X \cdots \times X$ by means of $(X)^{n}$, where $X$ is a normed vector space.

Consider the multivalued Nemytskii operator generated by $\Psi$, that is the operator $\mathcal{N}_{\Psi}:\left(L^{6}\left(Q_{T}\right)\right)^{2} \multimap\left(L^{2}\left(Q_{T}\right)\right)^{3}$ given by

$$
\begin{aligned}
\mathcal{N}_{\Psi}(p, q)(x, t):= & \left\{(f, g, h) \in\left(L^{2}\left(Q_{T}\right)\right)^{3}:(f(x, t), g(x, t), h(x, t))\right. \\
& \in \Psi\left(x, t, p(x, t), q(x, t), \phi_{1}(p)(t), \phi_{2}(q)(t), \phi_{3}(p)(t), \phi_{4}(q)(t)\right) \\
& \text { for a.e. } \left.(x, t) \in Q_{T}\right\},
\end{aligned}
$$

where $\phi_{i}(w)(t)=\int_{\Omega} K_{i}\left(x, t-\tau_{i}\right) w^{2}(x, t) d x, i=1,2,3,4$, and $t \in[0, T]$.

For the multivalued Nemytskii operator $\mathcal{N}_{\Psi}$ we have the following result.

Lemma 4.1 (see [16], Theorem 5.1). The multivalued map $\mathcal{N}_{\Psi}$ has bounded closed convex values and maps bounded sets into bounded sets. Moreover,

$$
\mathcal{N}_{\Psi}(p, q)=\bigcup_{(a, b) \in U} \Psi_{a, b}(p, q)
$$

for any $(p, q) \in\left(L^{6}\left(Q_{T}\right)\right)^{2}$, where

$$
\Psi_{a, b}(p, q)(x, t)=\psi_{a, b}\left(x, t, p(x, t), q(x, t), \phi_{1}(p)(t), \phi_{2}(q)(t), \phi_{3}(p)(t), \phi_{4}(q)(t)\right) .
$$

In order to solve the optimization problem first of all we prove a relevant property concerning the solution map $\mathcal{G}$ of the following Cauchy problems with Dirichlet boundary conditions.

$$
\left\{\begin{array}{l}
\frac{\partial u}{\partial t}-\Delta u^{m}=f \\
\frac{\partial v}{\partial t}-\Delta v^{m}=g, \\
\left.u(\cdot, t)\right|_{\partial \Omega}=\left.v(\cdot, t)\right|_{\partial \Omega}=0, \quad \text { for a.a. } t \in(0, T), \\
u(\cdot, 0)=u^{0}(\cdot), \\
v(\cdot, 0)=v^{0}(\cdot),
\end{array}\right.
$$

and

$$
\left\{\begin{array}{l}
\dot{w}(t)=\int_{\Omega} h(x, t) d x, \quad \text { a.e. in }[0, T], \\
w(0)=0 .
\end{array}\right.
$$

The assumptions on the data $f, g, h, u^{0}, v^{0}$ will be precised later, for the moment we assume only their integrability to permit the formulation of the following definition of weak solution.

Definition 4.1. ([21], Definition 5.4, p.87). By a solution $(u, v)$ of (28) we mean locally integrable functions $u, v$ defined in $Q_{T}$ such that

1. $u, v \in L^{1}\left(Q_{T}\right)$ and $u^{m}, v^{m} \in L^{1}\left((0, T), W^{1,1}(\Omega)\right)$, 
2. $u$ and $v$ satisfy the identities

$$
\iint_{Q_{T}}-u \frac{\partial \varphi}{\partial t}+\nabla u^{m} \nabla \varphi=\int_{\Omega} u^{0}(x) \varphi(x, 0) d x+\iint_{Q_{T}} f \varphi d x d t
$$

and

$$
\iint_{Q_{T}}-v \frac{\partial \varphi}{\partial t}+\nabla v^{m} \nabla \varphi=\int_{\Omega} v^{0}(x) \varphi(x, 0) d x+\iint_{Q_{T}} g \varphi d x d t
$$

for any $\varphi \in C^{1}\left(\bar{Q}_{T}\right)$, such that $\varphi(x, t)=0$ for all $(x, t) \in \partial \Omega \times[0, T]$ and $\varphi(x, T)=0$ for any $x \in \Omega$.

For our purposes we consider $f, g, h \in L^{2}\left(Q_{T}\right)$ and $u^{0}, v^{0} \in L^{\infty}(\Omega)$. Under these conditions by means of ([21], Theorem 5.7, p.97, and Theorem 6.2, p.128) there exists a unique weak solution $(u, v)$ of $(28)$, which is also a limit solution of classical solutions, with the properties: $u, v \in L^{\infty}\left((0, T), L^{2}(\Omega)\right)$ and $u^{m}, v^{m} \in$ $L^{2}\left((0, T), H_{0}^{1}(\Omega)\right)$. Therefore, the solution map $\mathcal{G}:\left(L^{2}\left(Q_{T}\right)\right)^{3} \times\left(L^{\infty}(\Omega)\right)^{2} \rightarrow$ $\left(L^{2}\left(Q_{T}\right)\right)^{2} \times L^{2}(0, T)$ given by

$$
\mathcal{G}\left(f, g, h, u^{0}, v^{0}\right)=(u, v, w)
$$

if and only if $(u, v)$ is the unique weak solution of (28) and $w$ is the solution of (29), is well defined.

Let $\mathcal{N}_{\Psi}^{0}:\left(L^{6}\left(Q_{T}\right)\right)^{2} \times\left(L^{\infty}(\Omega)\right)^{2} \multimap\left(L^{2}\left(Q_{T}\right)\right)^{3} \times\left(L^{\infty}(\Omega)\right)^{2}$ be the operator defined by

$$
\mathcal{N}_{\Psi}^{0}\left(p, q, u^{0}, v^{0}\right)=\left(\mathcal{N}_{\Psi}(p, q), u^{0}, v^{0}\right)
$$

We have the following result.

Proposition 4.1. The operator $\mathcal{G} \circ \mathcal{N}_{\Psi}^{0}:\left(L^{6}\left(Q_{T}\right)\right)^{2} \times\left(L^{\infty}(\Omega)\right)^{2} \multimap\left(L^{2}\left(Q_{T}\right)\right)^{2} \times$ $L^{2}(0, T)$ has closed graph.

Proof. Consider arbitrary sequences $\left(p_{n}, q_{n}\right) \in\left(L^{6}\left(Q_{T}\right)\right)^{2},\left(y_{n}^{0}, z_{n}^{0}\right) \in\left(L^{\infty}(\Omega)\right)^{2}$ and $\left(y_{n}, z_{n}, w_{n}\right) \in\left(L^{2}\left(Q_{T}\right)\right)^{2} \times L^{2}(0, T)$ so that $\left(y_{n}, z_{n}, w_{n}\right) \in \mathcal{G}\left(\mathcal{N}_{\Psi}^{0}\left(p_{n}, q_{n}, y_{n}^{0}, z_{n}^{0}\right)\right)$ with $\left(p_{n}, q_{n}\right),\left(y_{n}^{0}, z_{n}^{0}\right)$ and $\left(y_{n}, z_{n}, w_{n}\right)$ converging respectively to $\left(p_{0}, q_{0}\right),\left(y^{0}, z^{0}\right)$ and $\left(y_{0}, z_{0}, w_{0}\right)$ as $n \rightarrow+\infty$. Since $\left(y_{n}, z_{n}, w_{n}\right) \in \mathcal{G}\left(\mathcal{N}_{\Psi}^{0}\left(p_{n}, q_{n}, y_{n}^{0}, z_{n}^{0}\right)\right)$, there exists a sequence $\left(f_{n}, g_{n}, h_{n}\right)$ with $\left(f_{n}, g_{n}, h_{n}, y_{n}^{0}, z_{n}^{0}\right) \in \mathcal{N}_{\Psi}^{0}\left(p_{n}, q_{n}, y_{n}^{0}, z_{n}^{0}\right)$ such that $\left(y_{n}, z_{n}, w_{n}\right)=\mathcal{G}\left(f_{n}, g_{n}, h_{n}, y_{n}^{0}, z_{n}^{0}\right)$, namely $\left(y_{n}, z_{n}, w_{n}\right)$ is the unique weak solution of the problem

$$
\left\{\begin{array}{l}
\frac{\partial y}{\partial t}-\Delta y^{m}=f_{n} \\
\frac{\partial z}{\partial t}-\Delta z^{m}=g_{n}, \\
\left.y(\cdot, t)\right|_{\partial \Omega}=\left.z(\cdot, t)\right|_{\partial \Omega}=0, \quad \text { for a.a. } t \in(0, T) \\
y(\cdot, 0)=y_{n}^{0}(\cdot), \\
z(\cdot, 0)=z_{n}^{0}(\cdot)
\end{array}\right.
$$




$$
\left\{\begin{array}{l}
\dot{w}(t)=\int_{\Omega} h_{n}(x, t) d x, \quad \text { a.e. in }[0, T], \\
w(0)=0
\end{array}\right.
$$

where $h_{n}(x, t) \in \mathcal{F}\left(x, t, y_{n}(x, t), z_{n}(x, t)\right):=\left\{F\left(x, t, y_{n}(x, t), z_{n}(x, t), \gamma_{1}, \gamma_{2}\right)\right.$ : $\left.\gamma_{1}, \gamma_{2} \in\left[\mu_{1}+\delta, c\right]\right\}$, for a.a. $(x, t) \in Q_{T}$.

Since $\mathcal{N}_{\Psi}$ sends bounded sets into bounded sets, by passing to a subsequence if necessary, we have that $\left(f_{n}, g_{n}, h_{n}\right)$ weakly converges to some $\left(f_{0}, g_{0}, h_{0}\right) \in$ $\left(L^{2}\left(Q_{T}\right)\right)^{3}$. We want to show that

$$
\left(y_{0}, z_{0}, w_{0}\right)=\lim _{n \rightarrow+\infty} \mathcal{G}\left(f_{n}, g_{n}, h_{n}, y_{n}^{0}, z_{n}^{0}\right)=\mathcal{G}\left(f_{0}, g_{0}, h_{0}, y^{0}, z^{0}\right) .
$$

To this end, let $\hat{w}_{0} \in L^{2}(0, T)$ be the solution of

$$
\left\{\begin{array}{l}
\dot{w}(t)=\int_{\Omega} h_{0}(x, t) d x, \quad \text { a.e. in }[0, T], \\
w(0)=0 .
\end{array}\right.
$$

Then $\hat{w}_{0}=w_{0}$. In fact, using the fact that $h_{n}$ weakly converges to $h_{0}$ in $L^{2}\left(Q_{T}\right)$, we have

$$
\mu_{n}(t):=\left|\int_{0}^{t} \int_{\Omega}\left(h_{n}(x, \tau)-h_{0}(x, \tau)\right) d x d \tau\right|^{2} \rightarrow 0, \quad \text { as } n \rightarrow+\infty,
$$

for a.e. $t \in(0, T)$. Moreover, since $h_{n}$ is bounded in $L^{\infty}\left(Q_{T}\right)$ we have that $0 \leq \mu_{n}(t) \leq C$, for a.e. $t \in[0, T]$. Thus we can apply Lebesgue's Theorem and we can conclude that

$$
\int_{0}^{T}\left|w_{n}(t)-\hat{w}_{0}(t)\right|^{2} d t=\int_{0}^{T}\left|\int_{0}^{t} \int_{\Omega}\left(h_{n}(x, \tau)-h_{0}(x, \tau)\right) d x d \tau\right|^{2} d t=\int_{0}^{T} \mu_{n}(t) d t \rightarrow 0,
$$

as $n \rightarrow+\infty$. On the other hand, by assumption, $\lim _{n \rightarrow+\infty}\left\|w_{n}-w_{0}\right\|_{L^{2}(0, T)}=0$, hence $\hat{w}_{0}=w_{0}$.

Furthermore, by ([5, Theorem 6.4, p. 89]), if $\left(\hat{y}_{0}, \hat{z}_{0}\right)$ is a weak solution of the problem

$$
\left\{\begin{array}{l}
\frac{\partial y}{\partial t}-\Delta y^{m}=f_{0}, \\
\frac{\partial z}{\partial t}-\Delta z^{m}=g_{0}, \\
\left.y(\cdot, t)\right|_{\partial \Omega}=\left.z(\cdot, t)\right|_{\partial \Omega}=0, \quad \text { for a.a. } t \in(0, T), \\
y(\cdot, 0)=y^{0}(\cdot), \\
z(\cdot, 0)=z^{0}(\cdot),
\end{array}\right.
$$


then, for $t \in[0, T]$, we have that

$$
\begin{aligned}
\left\|y_{n}(\cdot, t)-\hat{y}_{0}(\cdot, t)\right\|_{L^{2}(\Omega)} & -\left\|y_{n}(\cdot, 0)-\hat{y}_{0}(\cdot, 0)\right\|_{L^{2}(\Omega)}+\left\|z_{n}(\cdot, t)-\hat{z}_{0}(\cdot, t)\right\|_{L^{2}(\Omega)}- \\
& -\left\|z_{n}(\cdot, 0)-\hat{z}_{0}(\cdot, 0)\right\|_{L^{2}(\Omega)} \\
& \leq \int_{0}^{t} \int_{\Omega}\left(y_{n}(x, \tau)-\hat{y}_{0}(x, \tau)\right)\left(f_{n}(x, \tau)-f_{0}(x, \tau)\right) d x d \tau+ \\
& +\int_{0}^{t} \int_{\Omega}\left(z_{n}(x, \tau)-\hat{z}_{0}(x, \tau)\right)\left(g_{n}(x, \tau)-g_{0}(x, \tau)\right) d x d \tau .
\end{aligned}
$$

Since $y_{n}$ converges to $y_{0}$ in $L^{2}\left(Q_{T}\right)$ and $f_{n}$ weakly converges to $f_{0}$ in $L^{2}\left(Q_{T}\right)$, as $n \rightarrow+\infty$, we have that

$$
\begin{aligned}
& \int_{0}^{t} \int_{\Omega}\left(y_{n}(x, \tau)-\hat{y}_{0}(x, \tau)\right)\left(f_{n}(x, \tau)-f_{0}(x, \tau)\right) d x d \tau \\
& \leq \int_{0}^{t} \int_{\Omega}\left(y_{n}(x, \tau)-y_{0}(x, \tau)+y_{0}(x, \tau)-\hat{y}_{0}(x, \tau)\right)\left(f_{n}(x, \tau)-f_{0}(x, \tau)\right) d x d \tau \\
& =\int_{0}^{t} \int_{\Omega}\left(y_{n}(x, \tau)-y_{0}(x, \tau)\right)\left(f_{n}(x, \tau)-f_{0}(x, \tau)\right) d x d \tau+ \\
& +\int_{0}^{t} \int_{\Omega}\left(y_{0}(x, \tau)-\hat{y}_{0}(x, \tau)\right)\left(f_{n}(x, \tau)-f_{0}(x, \tau)\right) d x d \tau \rightarrow 0,
\end{aligned}
$$

as $n \rightarrow+\infty$. Analogously,

$$
\lim _{n \rightarrow+\infty} \int_{0}^{t} \int_{\Omega}\left(z_{n}(x, \tau)-\hat{z}_{0}(x, \tau)\right)\left(g_{n}(x, \tau)-g_{0}(x, \tau)\right) d x d \tau=0 .
$$

Moreover, $\left\|y_{n}(\cdot, 0)-\hat{y}_{0}(\cdot, 0)\right\|_{L^{2}(\Omega)}$ and $\left\|z_{n}(\cdot, 0)-\hat{z}_{0}(\cdot, 0)\right\|_{L^{2}(\Omega)}$ tend to 0 as $n \rightarrow \infty$, hence, for $t \in[0, T]$, we have that

$$
\lim _{n \rightarrow+\infty}\left\|y_{n}(\cdot, t)-\hat{y}_{0}(\cdot, t)\right\|_{L^{2}(\Omega)}=\lim _{n \rightarrow+\infty}\left\|z_{n}(\cdot, t)-\hat{z}_{0}(\cdot, t)\right\|_{L^{2}(\Omega)}=0 .
$$

Thus, $\left(\hat{y}_{0}, \hat{z}_{0}, \hat{w}_{0}\right)=\left(y_{0}, z_{0}, w_{0}\right)$ and we can conclude that

$$
\lim _{n \rightarrow+\infty} \mathcal{G}\left(f_{n}, g_{n}, h_{n}, y_{n}^{0}, z_{n}^{0}\right)=\mathcal{G}\left(f_{0}, g_{0}, h_{0}, y^{0}, z^{0}\right) .
$$

Finally, since $\left(p_{n}, q_{n}\right)$ converges to $\left(p_{0}, q_{0}\right)$ in $\left(L^{6}\left(Q_{T}\right)\right)^{2}$ and $\left(y_{n}^{0}, z_{n}^{0}\right)$ converges to $\left(y^{0}, z^{0}\right)$ in $\left(L^{\infty}(\Omega)\right)^{2}$, we have, by [16, Theorem 5.1 - (ii)], that

$$
d_{\left(L^{2}\left(Q_{T}\right)\right)^{3} \times\left(L^{\infty}(\Omega)\right)^{2}}\left(\left(f_{n}, g_{n}, h_{n}, y_{n}^{0}, z_{n}^{0}\right), \mathcal{N}_{\Psi}^{0}\left(p_{0}, q_{0}, y^{0}, z^{0}\right)\right) \rightarrow 0,
$$

as $n \rightarrow+\infty$, where $d_{X}(a, A)$ denotes the distance in $X$ of $a \in X$ from the set $A \subset X$. On the other hand, from (32) and (33) we obtain that $\mathcal{G}:\left(L^{2}\left(Q_{T}\right)\right)^{3} \times$ $\left(L^{\infty}(\Omega)\right)^{2} \rightarrow\left(L^{2}\left(Q_{T}\right)\right)^{2} \times L^{2}(0, T)$ is continuous, thus

$$
d_{\left(L^{2}\left(Q_{T}\right)\right)^{2} \times L^{2}(0, T) \times\left(L^{\infty}(\Omega)\right)^{2}}\left(\mathcal{G}\left(f_{n}, g_{n}, h_{n}, y_{n}^{0}, z_{n}^{0}\right), \mathcal{G}\left(\mathcal{N}_{\Psi}^{0}\left(p_{0}, q_{0}, y^{0}, z^{0}\right)\right)\right) \rightarrow 0 .
$$


Therefore

$$
\left(y_{0}, z_{0}, w_{0}\right)=\mathcal{G}\left(f_{0}, g_{0}, h_{0}, y^{0}, z^{0}\right) \in \overline{\mathcal{G}\left(\mathcal{N}_{\Psi}^{0}\left(p_{0}, q_{0}, y^{0}, z^{0}\right)\right)}\left(L^{2}\left(Q_{T}\right)\right)^{2} \times L^{2}(0, T)
$$

The same arguments of the first part of the proof show that

$$
\overline{\mathcal{G}\left(\mathcal{N}_{\Psi}^{0}\left(p_{0}, q_{0}, y^{0}, z^{0}\right)\right)}\left(L^{2}\left(Q_{T}\right)\right)^{2} \times L^{2}(0, T)=\mathcal{G}\left(\mathcal{N}_{\Psi}^{0}\left(p_{0}, q_{0}, y^{0}, z^{0}\right)\right) .
$$

In fact, if one considers a sequence $\left(y_{n}, z_{n}, w_{n}\right) \in \mathcal{G}\left(\mathcal{N}_{\Psi}^{0}\left(p_{0}, q_{0}, y^{0}, z^{0}\right)\right)$ with $\left(y_{n}, z_{n}, w_{n}\right) \rightarrow\left(y_{0}, z_{0}, w_{0}\right)$ in $\left(L^{2}\left(Q_{T}\right)\right)^{2} \times L^{2}(0, T)$, as $n \rightarrow+\infty$, then there exists a sequence $\left(f_{n}, g_{n}, h_{n}\right) \in \mathcal{N}_{\Psi}\left(p_{0}, q_{0}\right)$ so that $\left(y_{n}, z_{n}, w_{n}\right)=\mathcal{G}\left(f_{n}, g_{n}, h_{n}, y^{0}, z^{0}\right)$. On the other hand, passing to a subsequence if necessary, we have that $\left(f_{n}, g_{n}, h_{n}\right)$ weakly converges in $\left(L^{2}\left(Q_{T}\right)\right)^{3}$ to some $\left(f_{0}, g_{0}, h_{0}\right) \in \mathcal{N}_{\Psi}\left(p_{0}, q_{0}\right)$, since $\mathcal{N}_{\Psi}\left(p_{0}, q_{0}\right)$ is a closed, convex set of $\left(L^{2}\left(Q_{T}\right)\right)^{3}$. This concludes the proof.

Now, we are in the position to prove the following result.

Theorem 4.1. The cost functional $J$ attains its minimum in $S$.

Proof. Let $\left(u_{n}, v_{n}, a_{n}, b_{n}\right) \in S$ be a minimizing sequence for the cost functional $J$; hence, by the existence results of the previous section, $\left(u_{n}, v_{n}\right) \in C\left(\bar{Q}_{T}\right) \times$ $C\left(\bar{Q}_{T}\right), u_{n}, v_{n} \geq 0$ in $Q_{T}$ and $\left\|u_{n}\right\|_{C\left(\bar{Q}_{T}\right)},\left\|v_{n}\right\|_{C\left(\bar{Q}_{T}\right)}>\lambda_{0}$, is a solution of (1) corresponding to $\left(a_{n}, b_{n}\right) \in U$. Now, set $u_{n}^{0}(\cdot):=u_{n}(\cdot, 0)=u_{n}(\cdot, T)$ and $v_{n}^{0}(\cdot):=v_{n}(\cdot, 0)=v_{n}(\cdot, T)$, hence $u_{n}^{0}, v_{n}^{0}$ are Hölder continuous in $\bar{\Omega}$ by the regularity results of [19], and consider the solution $w_{n} \in L^{2}(0, T)$ of the problem

$$
\left\{\begin{array}{l}
\dot{w}(t)=\int_{\Omega} h_{n}(x, t) d x, \quad \text { a.e. in }[0, T], \\
w(0)=0
\end{array}\right.
$$

where $h_{n}(x, t):=F\left(x, t, u_{n}(x, t), v_{n}(x, t), a_{n}(x, t), b_{n}(x, t)\right)$.

Then $\left(u_{n}, v_{n}, w_{n}\right) \in \mathcal{G}\left(\mathcal{N}_{\Psi}^{0}\left(u_{n}, v_{n}, u_{n}^{0}, v_{n}^{0}\right)\right)$, namely $w_{n}$ is defined by (34) and $\left(u_{n}, v_{n}\right)$ is a weak solution of the problem.

$$
\left\{\begin{aligned}
& \frac{\partial u}{\partial t}-\Delta u^{m}=\left(a_{n}(x, t)-\int_{\Omega} K_{1}(\xi, t) u^{2}\left(\xi, t-\tau_{1}\right) d \xi+\right. \\
&\left.+\int_{\Omega} K_{2}(\xi, t) v^{2}\left(\xi, t-\tau_{2}\right) d \xi\right) u \\
& \frac{\partial v}{\partial t}-\Delta v^{m}=\left(b_{n}(x, t)+\int_{\Omega} K_{3}(\xi, t) u^{2}\left(\xi, t-\tau_{3}\right) d \xi-\right. \\
&\left.-\int_{\Omega} K_{4}(\xi, t) v^{2}\left(\xi, t-\tau_{4}\right) d \xi\right) v \\
&\left.u(\cdot, t)\right|_{\partial \Omega}=\left.v(\cdot, t)\right|_{\partial \Omega}=0, \quad \text { for a.a. } t \in(0, T) \\
& u(\cdot, 0)=u_{n}^{0}(\cdot) \\
& v(\cdot, 0)=v_{n}^{0}(\cdot)
\end{aligned}\right.
$$


By the uniform boundedness of the functions $u_{n}, v_{n}, a_{n}$ and $b_{n}$ in $L^{\infty}\left(Q_{T}\right)$ it is easy to see that all the assumptions of [19, Theorem 1.2] are satisfied. Therefore as in the proof of Theorem 2.1, by passing to a subsequence, if necessary, $\left(u_{n}, v_{n}\right)$ converges uniformly in $\bar{Q}_{T}$ to a pair $\left(u_{0}, v_{0}\right) \in C\left(\bar{Q}_{T}\right) \times C\left(\bar{Q}_{T}\right)$, $\lambda_{0} \leq\left\|u_{0}\right\|_{C\left(\bar{Q}_{T}\right)},\left\|v_{0}\right\|_{C\left(\bar{Q}_{T}\right)} \leq R$. Furthermore, repeating the arguments of the proof of Proposition 4.1, one has that $w_{n}$ converges to $w_{0}$ in $L^{2}(0, T)$, where $w_{0}$ solves

$$
\left\{\begin{array}{l}
\dot{w}(t)=\int_{\Omega} h_{0}(x, t) d x, \quad \text { a.e. in }[0, T] \\
w(0)=0
\end{array}\right.
$$

being $h_{0}$ the weakly limit of $h_{n}$ in $L^{2}\left(Q_{T}\right)$. Furthermore, since $\mathcal{G} \circ \mathcal{N}_{\Psi}^{0}$ : $\left(L^{6}\left(Q_{T}\right)\right)^{2} \times\left(L^{\infty}(\Omega)\right)^{2} \multimap\left(L^{2}\left(Q_{T}\right)\right)^{2} \times L^{2}(0, T)$ has closed graph, we have that $\left(u_{0}, v_{0}, w_{0}\right) \in \mathcal{G}\left(\mathcal{N}_{\Psi}^{0}\left(u_{0}, v_{0}, u^{0}, v^{0}\right)\right)$. Thus, by $(27)$, there exists $\left(a_{0}, b_{0}\right) \in U$ such that

$$
\left(u_{0}, v_{0}, w_{0}\right)=\mathcal{G}\left(\Psi_{a_{0}, b_{0}}\left(u_{0}, v_{0}, u^{0}, v^{0}\right)\right),
$$

i.e. $\left(u_{0}, v_{0}\right)$ is a weak solution of the problem

$$
\left\{\begin{aligned}
& \frac{\partial u}{\partial t}-\Delta u^{m}=\left(a_{0}(x, t)-\int_{\Omega} K_{1}(\xi, t) u^{2}\left(\xi, t-\tau_{1}\right) d \xi+\right. \\
&\left.+\int_{\Omega} K_{2}(\xi, t) v^{2}\left(\xi, t-\tau_{2}\right) d \xi\right) u \\
& \frac{\partial v}{\partial t}-\Delta v^{m}=\left(b_{0}(x, t)+\int_{\Omega} K_{3}(\xi, t) u^{2}\left(\xi, t-\tau_{3}\right) d \xi-\right. \\
&\left.-\int_{\Omega} K_{4}(\xi, t) v^{2}\left(\xi, t-\tau_{4}\right) d \xi\right) v \\
&\left.u(\cdot, t)\right|_{\partial \Omega}=\left.v(\cdot, t)\right|_{\partial \Omega}=0, \quad \text { for a.a. } t \in(0, T) \\
& u(\cdot, 0)=u^{0}(\cdot) \\
& v(\cdot, 0)=v^{0}(\cdot)
\end{aligned}\right.
$$

and $w_{0}$ is such that

$$
\left\{\begin{array}{l}
\dot{w}(t)=\int_{\Omega} F\left(x, t, u_{0}(x, t), v_{0}(x, t), a_{0}(x, t), b_{0}(x, t)\right) d x, \quad \text { a.e. in } Q_{T}, \\
w(0)=0
\end{array}\right.
$$

Here $u^{0}$ and $v^{0}$ are the uniform limits of $u_{n}^{0}$ and $v_{n}^{0}$ in $\Omega$, respectively, thus they belong to $C(\bar{\Omega})$. Moreover, repeating the arguments of the proof of Theorem 2.1 with $\epsilon=0$, one can show that $u_{n}^{m}$ and $v_{n}^{m}$ are uniformly bounded in $L^{2}\left((0, T) ; H_{0}^{1}(\Omega)\right)$ and thus $u_{0}^{m}, v_{0}^{m} \in L^{2}\left((0, T) ; H_{0}^{1}(\Omega)\right)$ and $\left(u_{0}, v_{0}\right)$ satisfies $(30)-(31)$.

\section{References}

[1] W. Allegretto, C. Mocenni, A. Vicino, Periodic solutions in modelling lagoon ecological interactions, J. Math. Biol., 51 (2005), 367-388. 
[2] W. Allegretto, P. Nistri, Existence and optimal control for periodic parabolic equations with nonlocal term, IMA Journal of Mathematical Control and Information, 16 (1999), 43-58.

[3] W. Allegretto, D. Papini, Analysis of a Lagoon Ecological Model with Anoxic Crises and Impulsive Harvesting, to appear in the special issue "Mathematical Methods and Modeling of Biophysical Phenomena" of Math. Comput. Modelling.

[4] M. Badii, Periodic solutions for a class of degenerate evolution problem, Nonlinear Analysis TMA, 44 (2001), 499-508.

[5] Ph. Bénilan, M. G. Crandall, A. Pazy, Evolution Equations Governed by Accretive Operators, Book in preparation.

[6] A. Calsina, C. Perello, Equations for biological evolution, Proc. Royal Soc. Edin., 125 A (1995), 939 - 958.

[7] A. Calsina, C. Perello, J. Saldana, Non-local reaction-diffusion equations modelling predator-prey coevolution, Publications Matematiques, 32 (1994), 315-325.

[8] E. DiBenedetto, "Degenerate Parabolic Equations", Springer-Verlag, New York, 1993.

[9] G. Fragnelli, P. Martinez, J. Vancostenoble, Qualitative properties of a population dynamics system describing pregnancy, Mathematical Models and Methods in Applied Sciences, 15 (2005), no. 4, 507-554.

[10] E. Gurtin, R.C. McCamy, On the diffusion of biological populations, Math. Biosc. 33 (1977), 35-49.

[11] E. Gurtin, R.C. McCamy, Diffusion models for age-structured populations, Math. Biosc. 54 (1981), 49-59.

[12] P. Hess, "Periodic-parabolic boundary value problems and positivity", Pitman Research Notes 247, John Wiley, New York, 1991.

[13] P. Hess, M.A. Pozio, A. Tesei, Time periodic solutions for a class of degenerate parabolic problems, Houston J. Math. 21 (1995), no. 2, 367-394.

[14] R. Huang, Y. Wang, Y. Ke, Existence of non-trivial nonnegative periodic solutions for a class of degenerate parabolic equations with nonlocal terms, Discrete and Continuous Dynamical Systems-Series B, 5 (2005), no. 4, 1005-1014.

[15] O. Ladyzenskaja, V. Solonnikov, N. Uraltseva, "Linear and Quasilinear Equations of Parabolic Type, Translations of Mathematical Monographs", 23, American Mathematical Society, Providence, 1968. 
[16] J. M. Lasry, R. Robert, Analyse nonlinéaire multivoque, Cahiers Math. de la Décision, 7611, Université de Paris, Dauphine, 1990.

[17] M. Nakao, Periodic solutions of some nonlinear degenerate parabolic equations, J. Math. Anal. Appl., 104 (1984), 554-567.

[18] A. Okubo, "Diffusion and ecological problems: mathematical models", Biomathematics, 10, Springer, 1980.

[19] M.M. Porzio, V. Vespri, Hölder estimates for local solution of some double degenerate parabolic equation, J. Differential Equation, 103 (1993), 146178.

[20] M. Schigesada, K. Kawasaki, E. Teramoto, Spatial segregation of interacting species, J. Theoret. Biol., 79 (1979), 83-99.

[21] J. L. Vazquez, The Porous Medium Equation: Mathematical Theory, Oxford Mathematical Monographs, Ed. Oxford Science Publications, 2007.

[22] Y. Wang, J. Ying, Z. Wu, Periodic solutions of porous medium equations with weakly nonlinear sources, Northeast. Math. J., 16 (2000), no. 4, 475483.

[23] Y. Wang, Z. Wu, J. Yin, Time periodic solutions of a class of degenerate parabolic equations, Acta Math. Appl. Sinica (English Ser.), 16 (2000), 180-187. 